\title{
Feedforward Inhibition Allows Input Summation to Vary in Recurrent Cortical Networks
}

\author{
DDark H. Histed
}

DOI:http://dx.doi.org/10.1523/ENEURO.0356-17.2018

National Institute of Mental Health Intramural Program, National Institutes of Health, Bethesda, MD 20892

\begin{abstract}
Brain computations depend on how neurons transform inputs to spike outputs. Here, to understand input-output transformations in cortical networks, we recorded spiking responses from visual cortex (V1) of awake mice of either sex while pairing sensory stimuli with optogenetic perturbation of excitatory and parvalbumin-positive inhibitory neurons. We found that V1 neurons' average responses were primarily additive (linear). We used a recurrent cortical network model to determine whether these data, as well as past observations of nonlinearity, could be described by a common circuit architecture. Simulations showed that cortical input-output transformations can be changed from linear to sublinear with moderate ( 20\%) strengthening of connections between inhibitory neurons, but this change away from linear scaling depends on the presence of feedforward inhibition. Simulating a variety of recurrent connection strengths showed that, compared with when input arrives only to excitatory neurons, networks produce a wider range of output spiking responses in the presence of feedforward inhibition.
\end{abstract}

Key words: Cortex; mouse; network model; optogenetic; vision

\section{Significance Statement}

Brains are made up of neural networks that process information by receiving input activity and transforming those inputs into output activity. We use optogenetic manipulations in awake mice to expose how a transformation in a cortical network depends on internal network activity. Combining numerical simulations with the data uncovers that this transformation depends critically on feedforward inhibition - the fact that inputs to the cortex often make strong connections on both excitatory and inhibitory neurons.

\section{Introduction}

Neurons in the cerebral cortex receive thousands of synaptic inputs and transform those inputs into spike outputs. Input-output transformations can be characterized in single cells (measuring firing rate while injecting current to produce a, f-I curve; Koike et al., 1970; Connors et al., 1982; Destexhe and Paré, 1999), but network effects can dramatically alter input-output transforma-

Received October 17, 2017; accepted January 5, 2018; First published January 19, 2018

The authors declare no competing financial interests.

Author contributions: M.H.H. designed research, performed research, analyzed data, and wrote the paper.

This work was funded in part by the Intramural Research Program of the National Institute of Mental Health and by U01 NS090576 (BRAIN Initiative).

Acknowledgments: I thank Nicolas Brunel and Alessandro Sanzeni for discussion and comments; Lindsey Glickfeld, Alex Handler, and Oliver Mithoefer for assistance with neurophysiology; John Maunsell for support; and Bruno tions in vivo. For example, ongoing network activity can create supralinearities in neurons' input-output functions (Priebe and Ferster, 2008), strong network connectivity can create entirely linear input-output functions (van Vreeswijk and Sompolinsky, 1996; Brunel, 2000), and recurrent connections can amplify inhibition to produce sublinearity (Ahmadian et al., 2013).

Averbeck, Barry Richmond, Lex Kravitz, and Carson Chow for comments on this manuscript.

Correspondence should be addressed to Mark $\mathrm{H}$. Histed, National Institute of Mental Health, National Institutes of Health, 35 Convent Dr. 35/3A-203, Bethesda, MD 20892. E-mail: mark.histed@nih.gov.

DOI:http://dx.doi.org/10.1523/ENEURO.0356-17.2018

Copyright (C) 2018 Histed

This is an open-access article distributed under the terms of the Creative Commons Attribution 4.0 International license, which permits unrestricted use, distribution and reproduction in any medium provided that the original work is properly attributed. 
In this work, we examine input-output transformations in vivo by first measuring spiking responses to combinations of visual and optogenetic input in the mouse visual cortex (V1). Then, to shed light on the network and circuit mechanisms of input-output transformations, we use a spiking recurrent network model. The experimental data show that excitatory neuron stimulation gives a primarily linear (additive) input-output transformation in mouse V1, which stands in contrast to sublinearity seen in monkey V1 (Nassi et al., 2015). The model shows that the cortical network can achieve both kinds of transformations with only moderate changes in local recurrent synaptic strengths. The model makes a further prediction that feedforward inhibition-input that synapses not just on excitatory but also on inhibitory neurons-allows the cortex to support both kinds of transformations.

Optogenetic stimulation can reveal how networks in vivo transform inputs into output. Studies using sensory stimuli alone are complicated by the fact sensory stimuli are processed by many brain regions, each of which may provide input to a cortical area under study. Combinations of sensory stimuli, however, have found that a wide range of transformations are possible, often finding evidence for normalization, a form of sublinear summation (Carandini and Heeger, 2012). A few recent studies have used direct optogenetic input to study input-output transformations, and studies in different species have observed both normalization (Sato et al., 2014; Nassi et al., 2015) and more linear summation (Huang et al., 2014), pointing to the need to understand what features of cortical networks can change input-output transformations.

Models and theoretical approaches complement experimental studies of input-output transformations, because is difficult to control connectivity in an in vivo cortical network experimentally. Rate-based models (Ahmadian et al., 2013; Rubin et al., 2015) have characterized the range of behaviors cortical networks can support. But not all the effects seen in rate-based models may occur in biological networks, as spiking neurons have biophysical properties that can impact input-output transformations, such as refractory periods and nonlinearities due to spike threshold. Analysis of networks of spiking neurons is most advanced for models that approximate neuronal inputs as currents and not conductances (e.g., Brunel, 2000), but input-output relationships can be modified by the changes in effective synaptic strength and $V_{m}$ variability (Richardson, 2004, 2007) that occur in realistic conductancebased neurons. Therefore, we use numerical simulations of models of conductance-based spiking neurons to determine which connectivity properties might create the input-output transformations seen in my data and in past data.

Below, we first describe the experimental results from excitatory optogenetic perturbations in mouse visual cortex (Figs. 1 and 2), showing near-linear responses across a wide range of firing rates and visual contrast. We then describe results from the model, showing that feedforward inhibition can produce sublinearity (Fig. 3), and that with feedforward inhibition, local connectivity can allow networks to be either linear or sublinear (Figs. 4 and 5).
Finally, we construct a model network (Fig. 6) that fits the observations and show it is consistent with data from optogenetic perturbations of inhibitory neurons (Fig. 7). The observations are together best described by a model with feedforward inhibition.

\section{Materials and Methods}

\section{Neurophysiology}

All animal procedures were performed in accordance with the Harvard Medical School animal care committee's regulations, and in accordance with $\mathrm{NIH}$ standards. A detailed description of the neurophysiological methods is given in Glickfeld et al. (2013). In brief: Neurophysiological data from Emx1-Cre animals ( $n=4$, of both sexes but sex not recorded) were collected. Animals kept on a monitored water schedule were given small drops of water $(\sim 1$ $\mu l)$ every $60-120 \mathrm{~s}$ during recording to keep them awake and alert. The visual stimulus, a Gabor patch with spatial frequency 0.1 cycle/deg and sigma $12.5 \mathrm{deg}$, were presented for $115 \mathrm{~ms}$ [full width at half maximum (FWHM) intensity], and successive visual stimuli were presented every $1 \mathrm{~s}$. Optogenetic light pulses were delivered on alternating sets of 10 stimulus presentations (light onset $500 \mathrm{~ms}$ before first stimulus; offset $500 \mathrm{~ms}$ after end of last stimulus; total light pulse duration $10.2 \mathrm{~s}$ ). A 1-s delay was added after each set of 10 stimulus presentations. Extracellular probes were 32-site silicon electrodes (Neuronexus, probe model $\mathrm{A} 4 \times 8$ ). Recording surfaces were treated with poly(3,4-ethylenedioxithiophene) (PEDOT) to lower impedance and improve recording quality. On each recording day, electrodes were introduced through the dura and left stationary for $\sim 1 \mathrm{~h}$ before recording to give more stable recordings. Channelrhodpsin-2 (ChR2) was expressed in excitatory neurons (as described in Histed and Maunsell, 2014) using viral (AAV-EF1a-DIO-ChR2mCherry, serotype 2 or 8 ; http://openoptogenetics.org) injections into the Emx1-Cre (Gorski et al., 2002; \#5628, Jackson Laboratory) line. Virus $(0.25-1.0 \mu \mathrm{l})$ was injected into a cortical site whose retinotopic location was identified by imaging autofluorescence responses to small visual stimuli. Light powers used for optogenetic stimulation were $500 \mu \mathrm{W} / \mathrm{mm}^{2}$ on the first recording session; in later sessions, dural thickening was visible and changes in firing rate were smaller, so power was increased (maximum $3 \mathrm{~mW} / \mathrm{mm}^{2}$ ) to give mean spontaneous rate increases of approximately $\sim 5 \mathrm{spk} / \mathrm{s}$ in that recording session. Optogenetic light spot diameter was 400-700 $\mu \mathrm{m}(\mathrm{FWHM})$ as measured by imaging the delivered light on the cortical surface.

Spike waveforms were sorted after the experiment using OfflineSorter (Plexon, Inc.). Single units (SU) were identified as waveform clusters that showed clear and stable separation from noise and other clusters, unimodal width distributions, and interspike interval histograms consistent with cortical neuron absolute and relative refractory periods. Multiunits (MU) were clusters that were distinct from noise but did not meet one or more of those criteria, and thus these multiunits likely group together a small number of single neuron waveforms. Signal-tonoise ratios (SNRs) of unit waveforms (Kelly et al., 2007) 
were median 4.57 for single units $(n=50)$ and 3.02 for multiunits $(n=239)$. Although SNR can depend on statistical clustering criteria, it allows comparison between units within a study and can allow approximate comparisons across studies. The SNRs we measured are similar to those from other multielectrode recordings (e.g., using "Utah" arrays; Wissig and Kohn (2012) saw median SNR across all units of $\sim 2.75$ ). The number of short interspike intervals was also lower in single units than multiunits, as expected due to neurons' refractory periods [ISIs $<2.5$ ms: SU 0.28\%, MU 1.2\%, $p<0.001$, Kolmogorov-Smirnov (KS) test; ratio of ISIs $<2.5 \mathrm{~ms}$ observed to those expected for a Poisson process with same rate: SU 0.26 , MU $0.72, p<0.001$, KS test]. We also repeated the singleand multiunit comparisons (Figs. 1 and 2) using both moreand less-stringent criteria to qualify a unit as a single unit and found no qualitative differences in the results.

\section{Experimental design and statistical analysis}

Spike histograms were smoothed using piecewise splines (LOWESS smoothing). To compute neurons' visual responses (e.g., Figs. $1 D$ and $2 A$ ), we counted spikes over a 175-ms period beginning $25 \mathrm{~ms}$ after stimulus onset, with a matched baseline period $175 \mathrm{~ms}$ long, ending at stimulus onset. To test for nonlinearity, for each cell we found the response count with and without optogenetic stimulation by taking the stimulus response count and subtracting the baseline count. Neurons were classified as significantly nonlinear if the $p$-value of a twosample two-tailed KS test on the counts with and without stimulation was $<0.01$. The $E m \times 1$ dataset includes data from 100 shank penetrations ( 25 recording sessions with a 4-shank electrode). Because the intershank spacing was $200-400 \mu \mathrm{m}$, our stimuli in fixed retinotopic locations could not activate neurons on all shanks. Therefore, we included only shanks in which an average visual response $>0.2 \mathrm{spk} / \mathrm{s}$ was measured ( $38 / 100$ shanks). This gave 417 single and multiunits. We examined only units that showed a visual stimulus response $(n=289$; mean stimulus response - mean spontaneous $>0.2$ ) in the absence of ChR2 stimulation. Because ChR2 expression was highest at the site of viral injection and fell off with distance, we took advantage of this variation to sort units into three groups based on the strength of local ChR2 activation (Fig. 1C). We found the average change in spontaneous rate induced by ChR2 stimulation for all units on a shank and rank-ordered the shanks. Dividing shanks into three groups based on small, medium, or large ChR2 effects yielded three nearly equal-sized groups of units receiving small, medium, or large ChR2 activation. The group sizes differ by a few units because we sorted by shank, not by individual unit.

Recording site depth was measured as the distance below the most superficial site at which activity was seen. More units were recorded at superficial than deep locations, in part because probe insertion was stopped after units were seen at the most superficial site on one shank. Small cortical curvature could thus lead to some shanks with no units at the deeper sites.

\section{Conductance-based spiking network model}

The cortical model is a recurrent network of conductancebased leaky integrate-and-fire neurons. Example Python code and a Jupyter notebook (http://jupyter.org) are provided at https://github.com/histedlab/code-feedforwardinhibition-condLIF. that run the network simulation with all its inputs, replicating spike counts shown in Fig. 6C, bottom row. To recover the rest of the simulations in Fig. $3-7$, this code can be run in parallel on a larger cluster.

Each model neuron is connected randomly to each other neuron with fixed probability (sparsity). For example, for a $10 \%$ sparsity network, each cell receives input from $10 \%$ of the excitatory cells and thus gets $0.1 \times 8000=$ $800 \mathrm{E}$ inputs. Similarly, at $10 \%$ sparsity, each cell receives $0.1 \times 2000=200 \mathrm{I}$ inputs. We chose the inhibitory synaptic strength to be larger than the excitatory synaptic strength, as seen in the cortex. We varied both synaptic strengths and found that our conclusions were not affected by changes in E/I synaptic strength ratio. (See also Fig. 5 for effects of changing together $E$ and I recurrent synaptic weights by an order of magnitude). We refer to this baseline set of random, sparse connections as the balancing connections. To change local connectivity, we change the strength of a second added set of connections with the same sparsity while keeping the strength of the balancing connections constant. For example, when $\mathrm{I} \rightarrow \mathrm{I}$ connectivity is varied in the $2 \%$ sparsity network (e.g., Fig. 4), each I cell receives an extra 40 synapses from other I cells, and the $y$-axis in Fig. $4 A, B$ shows the effects of varying the weight of those 40 synapses from zero to $\sim 20 \%$ of the weight of the standard recurrent $|\rightarrow|$ synapses.

Each simulated neuron's membrane potential evolves according to the following equation:

$$
\begin{gathered}
\frac{d V_{m}}{d t}=-\frac{1}{\tau_{m}}\left[g_{\text {leak }}\left(V_{m}-E_{\text {rest }}\right)+g_{C h R 2}\left(V_{m}-E_{e}\right)+g_{e}\right. \\
\left.\left(V_{m}-E_{e}\right)+g_{i}\left(V_{m}-E_{i}\right)\right] .
\end{gathered}
$$

When the membrane potential $V_{m}$ crosses a threshold $(-50 \mathrm{mV})$, a spike is recorded and $V_{m}$ is reset to $E_{\text {rest }}(-60$ $\mathrm{mV}$ ) for the absolute refractory period (3 ms).

Beyond the recurrent inputs from other neurons in the network (described in the model architecture above), model neurons can receive two kinds of external inputs: external feedforward inputs simulating, e.g., sensory input from thalamus, and external ChR2 inputs. Feedforward (sensory) inputs are simulated as Poisson spike trains whose rates are changed by stepping to a new value, with values chosen to approximate visually-evoked changes seen in the data. ChR2 input is simulated by linearly ramping $g_{C h R 2}$ to a new value over $2 \mathrm{~ms}$, a timescale consistent with ChR2 $t_{\text {on }}$ (Nikolic et al., 2009), and $g_{C h R 2}$ amplitude is varied to reproduce experimental changes in firing rate (see below). Synaptic conductances $g_{e}$ and $g_{i}$ are incremented instantaneously by a constant excitatory or inhibitory synaptic weight when a spike is fired by a recurrent or feedforward input. The conductances decay with time constants $\tau_{g e}=5$ $\mathrm{ms}$ and $\tau_{g i}=10 \mathrm{~ms}$, described by 


$$
\begin{aligned}
& \frac{d g_{e}}{d t}=-\frac{g_{e}}{\tau_{g_{e}}}, \\
& \frac{d g_{i}}{d t}=-\frac{g_{i}}{\tau_{g_{i}}} .
\end{aligned}
$$

Other constants are: excitatory reversal $\left(E_{e}\right)=0 \mathrm{mV}$, inhibitory reversal $\left(E_{i}\right)=-80 \mathrm{mV}$, membrane time constant $\left(\tau_{m}\right)=20 \mathrm{~ms}$. (Because neurons are conductance based, effective time constants are shorter; Destexhe and Paré, 1999; Richardson, 2004.) Postsynaptic potential (PSP) amplitudes can vary with network activity and synaptic weight in the conductance-based model neurons. As we varied sparsity in the network, the excitatory PSP amplitude varied over an $\sim 10$-fold range $(0.3-3.0 \mathrm{mV}$ for sparsity $20 \%-2 \%$, if calculated assuming that the mean membrane potential of network neurons is $-65 \mathrm{mV}$.) Median $V_{m}$ varies across neurons of the network (during spontaneous activity without visual or ChR2 stimulation, 5th-95th percentile of neuron median $V_{m}:-71.0$ to $-60.5 \mathrm{mV}$ ).

The sparse recurrent connections yield spontaneous activity in the network in the absence of external input (van Vreeswijk and Sompolinsky, 1998; Vogels and Abbott, 2005). To equate the spontaneous firing state of the network across different sparsity and synaptic strength, we adjusted network spontaneous rate. Although several biophysical or input properties could be varied in neurons to change spontaneous rate, we used an additional external Poisson excitatory input to either E or I neurons to respectively raise or lower the spontaneous rate. The rate of this Poisson input was chosen via stepwise optimization to give a mean spontaneous rate across excitatory neurons of $5 \mathrm{spk} / \mathrm{s}$. The added input is less than the input coming from neurons within the network; e.g., in Fig. 4, across all points plotted for sparsities $2 \%-20 \%$ (rows 2, 4 , and 5), added input is $0.4 \%-18.9 \%$ (5th-90th percentile; maximum $69 \%$ ) of the total conductance input to the cell during spontaneous activity, and smaller when the network is driven by ChR2 or visual input. For many networks, a local minimum of the parameter can be found repeatably, but for extreme values of sparsity and synaptic strength, the network is unstable and spontaneous rates are either sensitive to small perturbations or diverge. In these cases, network response is not shown (e.g., gray regions, Fig. 5B,C).

Simulations were performed with the Brian package (Brette et al., 2007) on a multi-CPU cluster with an integration time step of $50 \mu \mathrm{s}$.

\section{Results}

\section{Experimental measurements in mouse V1 show linear summation}

We combined visual and excitatory optogenetic input (Fig. $1 A, B$ ) by expressing ChR2 in V1 excitatory neurons using a transgenic mouse line and a Cre-dependent virus, and we used blue light pulses several seconds in duration (4-6 s) to shift neurons' firing rates to a new baseline. We delivered the same visual stimulus repeatedly, with and without ChR2 stimulation. We kept animals alert by giving them drops of fluid approximately once a minute, and we measured neurons' spiking via extracellular recording with multisite probes.

When we presented the same visual stimulus with and without optogenetic stimulation, we found that V1 neurons' responses scaled nearly linearly (Fig. 1C) -that is, nearly the same size response was produced even as the optogenetic stimulus changed the baseline firing rate. Even for relatively large optogenetic baseline shifts $(\sim 10$ $\mathrm{spk} / \mathrm{s}$, roughly the same magnitude as the average visual response), the visual response was similar with and without ChR2 stimulation. This response implies that the input-output transformation is linear (also called additive, e.g., Huang et al., 2014), meaning the sensory response produces a fixed change in firing rate above the changing baseline rate. (In contrast, if the response were sublinear, higher baseline rates would produce a smaller sensory response.) We saw nearly linear responses across a range of intensities of the visual stimulus (contrast range $8 \%-$ $90 \%$; Fig. 1D), and we saw linear responses in both averages across single units $(n=50)$ and multiunits $(n=$ 239). Responses became slightly sublinear in cells with the largest baseline shifts (Fig. 1E), but responses were on average within a few percentage points of linear (for maximum contrast, as in Fig. 1D: average sensory response changed from 9.6 to $8.7 \mathrm{spk} / \mathrm{s}$, a $-9.5 \%$ change; for single units, $-7.9 \%$; for multiunits, $-10.0 \%$; in contrast, the average baseline rate almost doubled: $5.8-10.8 \mathrm{spk} / \mathrm{s}$; change $86 \%)$. This sublinearity is small compared with the normalization typically seen in visual cortex of species such as the macaque ( $~ 50 \%$ sublinearity seen when combining optogenetic and visual stimuli in V4; Nassi et al., 2015; as shown by their Fig. 4E,F; note that their additive index $=0.67$ is in our terminology approximately equal to a $50 \%$ sublinearity).

Firing rate variation could be an initial hypothesis to explain observed variation in scaling across studies. Consistent with this idea, responses of single neurons in macaque MT show linear scaling at low rate and sublinear scaling at high firing rate for combinations of visual stimuli (Britten and Heuer, 1999). Mouse V1 visual processing operates at lower firing rates than macaque $\mathrm{V} 1$, as strong (high-contrast) visual stimuli gave $\sim 10 \mathrm{spk} / \mathrm{s}$ average responses (Fig. 1), equal to or larger than previously reported (e.g., Adesnik et al., 2012) but much lower than the $100 \mathrm{spk} / \mathrm{s}$ responses at times seen in macaque V1 (Nassi et al., 2015). However, several experiments that combine optogenetic and visual stimulation argue that other mechanisms are in play besides just firing rate variation. In some cells of macaque V1, complete sublinearity is seen with combinations of optogenetic and visual input, even at low firing rates (Nassi et al., 2015; their Fig. 6B,F). And, in tree shrew V1 (Huang et al., 2014; e.g., their Fig. 7C,I), nearly linear responses are seen with somewhat higher firing rates than here. Although firing rate variation may partially explain the differences seen across animals, we wished to understand whether cortical summation can be dramatically affected by circuit properties such as recur- 
A

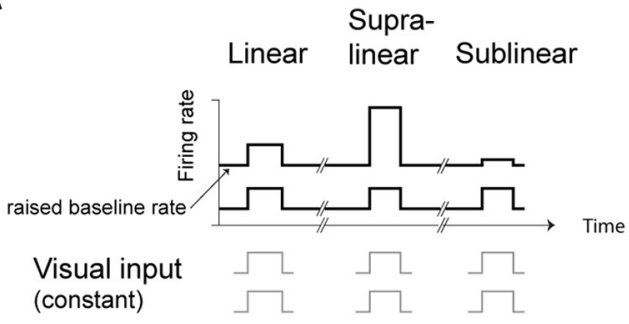

B

II. Same visual
stimulus repeated

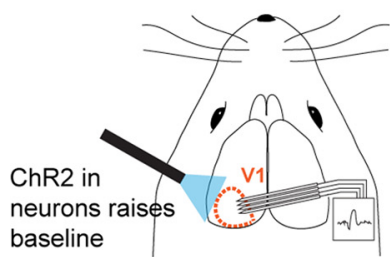

D
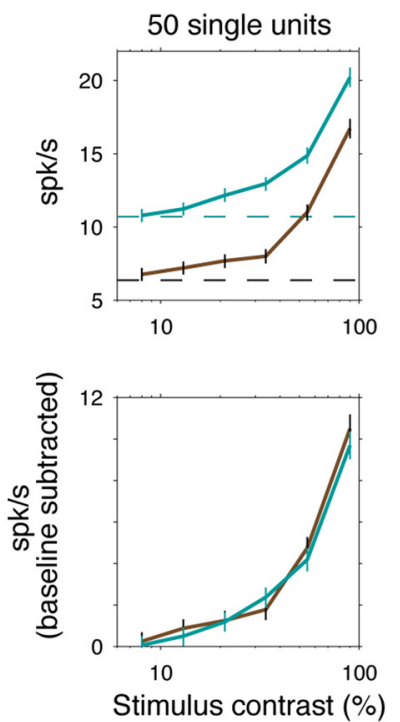

C
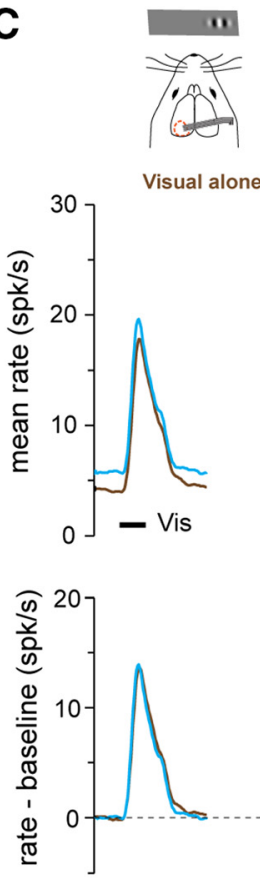

10

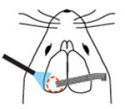

Visual

+ ChR2

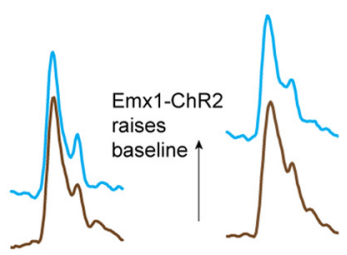

$200 \mathrm{~ms}$
E
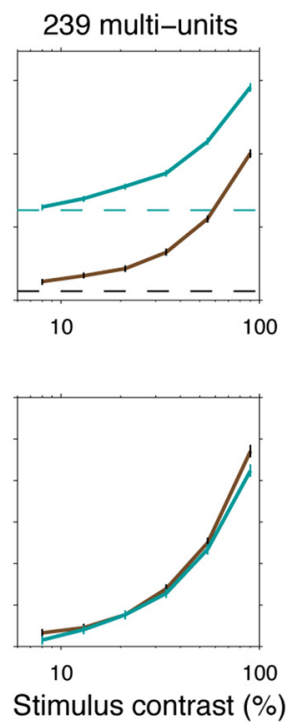
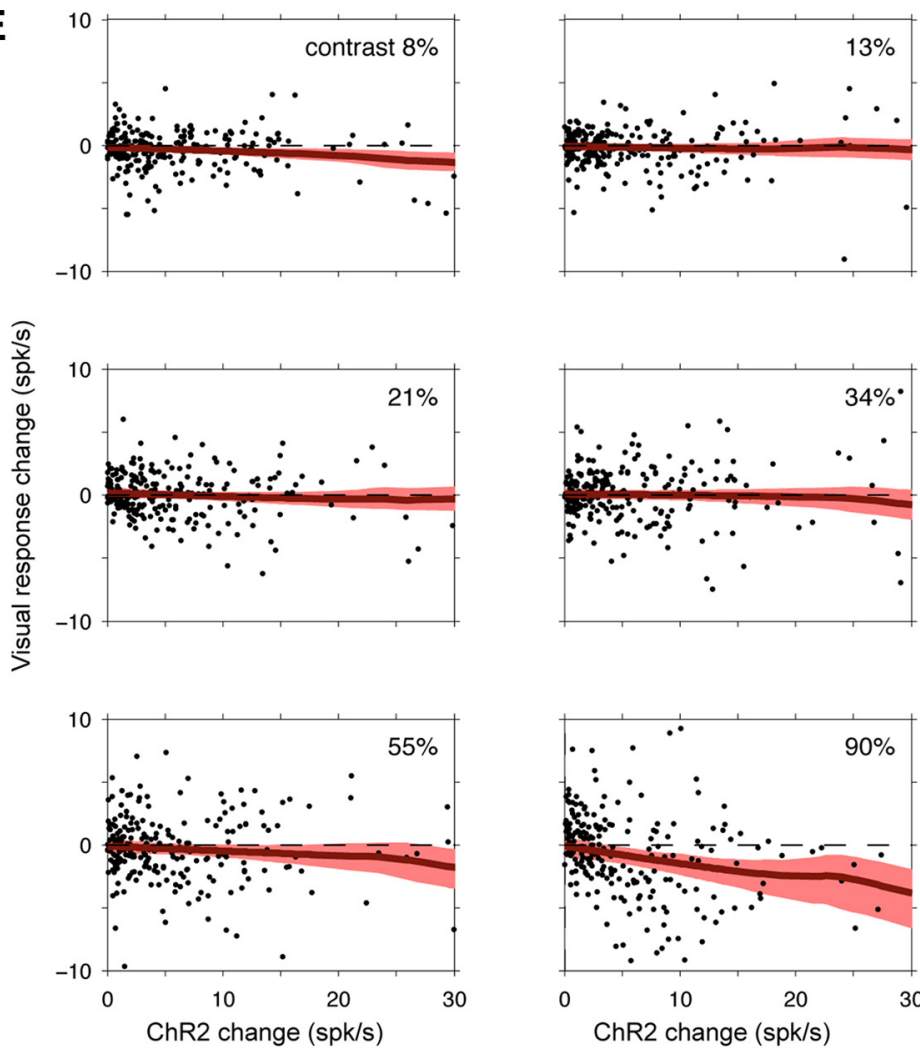

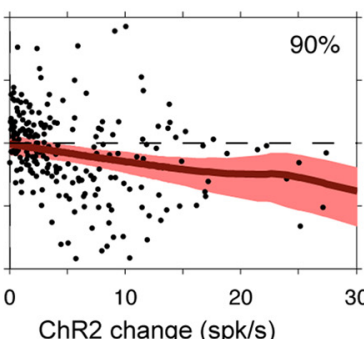

Figure 1. Near-linear scaling with excitatory optogenetic stimulation in mouse V1. $\boldsymbol{A}$, Schematic of experimental stimulus protocol. If scaling is linear, the same input pulse produces the same response when baseline (spontaneous) rate is changed. $\boldsymbol{B}$, Baseline rates are raised using ChR2 in excitatory (E) neurons (Cre-dependent virus in Emx1-Cre mouse line). $\boldsymbol{C}$, Population histograms showing responses to combined ChR2 and visual (90\% contrast) stimuli. In the top row, columns show three groups of neurons, divided based on size of ChR2 baseline firing rate changes: left, smallest ChR2 effects ( $n=94 ; 36$ single, 58 multiunits); middle, intermediate ChR2 effects ( $n=101$; 31 single, 70 multiunits); right, largest ChR2 effects ( $n=94 ; 28$ single, 66 multiunits). Brown, responses to visual stimulus with no optogenetic stimulus; cyan, responses to visual stimulus when baseline rates are changed by sustained optogenetic stimulus. The bottom row shows the same data as the top row, with spontaneous firing rates subtracted. Visual responses differ 
continued

somewhat between columns because each column is a different group of neurons, but within each group there is little response change as spontaneous rate varies. $\boldsymbol{D}$, Linear scaling is seen across a wide contrast range. Top row, responses without baseline subtraction; bottom row, baseline subtracted. Error bars are SEM of pooled unit responses. $\boldsymbol{E}$, Linear scaling is seen on average, across neurons with a variety of ChR2-induced baseline rate changes, with some weak sublinearity at the highest rate changes and highest contrasts. $Y$ axes, difference in visual responses (relative to baseline) with and without ChR2 stimulation; dashed line at zero shows a perfectly linear response. Red, LOWESS regression; shaded region is a bootstrapped $95 \%$ confidence interval. Two outlier points in $90 \%$ contrast plot are omitted for visual clarity although they are included in the regression; the two outliers are shown in Fig. $2 A$.

rent connectivity. Thus, below we use simulations to explore how network recurrent connectivity might change summation.

We observed that average neuronal responses were nearly linear, but individual recorded units were often either supra- or sublinear (Fig. 2). Units with large and small ChR2 effects are nonlinear (points lie above or below the horizontal line that shows a perfectly linear response; Fig. 2A). Both SU and MU are nonlinear (Fig. $2 A$; example time courses in Fig. 2B). With the $90 \%$ contrast visual stimulus, $34 \%$ of single units are significantly nonlinear (17/50, $p<0.01$, KS test; Fig. $2 A)$, and $28 \%$ of multiunits are significantly nonlinear $(67 / 239, p<$ 0.01 , KS test).

We examined whether cortical layer or depth might explain some of the observed heterogeneity in response scaling in different cells. To do this, we regressed percentage change in visual response (before and after ChR2 stimulation) on depth of recording site. We found that depth did not affect the amount of nonlinearity (coefficient not significantly different from zero, regression $p>0.70$ ). Site depth, however, did affect the magnitude of units' responses to ChR2, as expected due to cortical surface illumination, with more superficial sites showing larger effects (units at sites with depth $\leq 300 \mu \mathrm{m}, n=188$, median ChR2 response $3.4 \mathrm{spk} / \mathrm{s}$, top quartile ChR2 response $8.0 \mathrm{spk} / \mathrm{s}$; units at sites with depth $\geq 400 \mu \mathrm{m}, n=$ 101, median ChR2 response $1.5 \mathrm{spk} / \mathrm{s}$, top quartile 3.8 $\mathrm{spk} / \mathrm{s}$ ). To further explore factors that might explain the size of the nonlinearity, we used multivariate regression with several explanatory variables, including depth, visual response magnitude, baseline firing rate without ChR2 stimulation, and whether a unit was a single or multiunit. None of these factors, individually or together, significantly explained the percentage change in visual response (for full model with three factors, regression $p=$ 0.33; no submodel with a subset of the independent variables had a $p$-value $<0.33)$. Fig. $1 C, E$ shows that ChR2 response magnitude can slightly predict the size of the nonlinearity, and the regressions show that the other factors, besides ChR2 response magnitude, do not add any additional power to predict the size of the nonlinearity.

Although such heterogeneity in response scaling could in principle arise from different levels of ChR2 expression in different cells (as are produced by the AAV viral transfection; Watakabe et al., 2015), it seems likely that the amount of ChR2 input to a given cell does not explain all heterogeneity. First, if the amount of ChR2 expression predicted a unit's summation, one might expect a strong
A

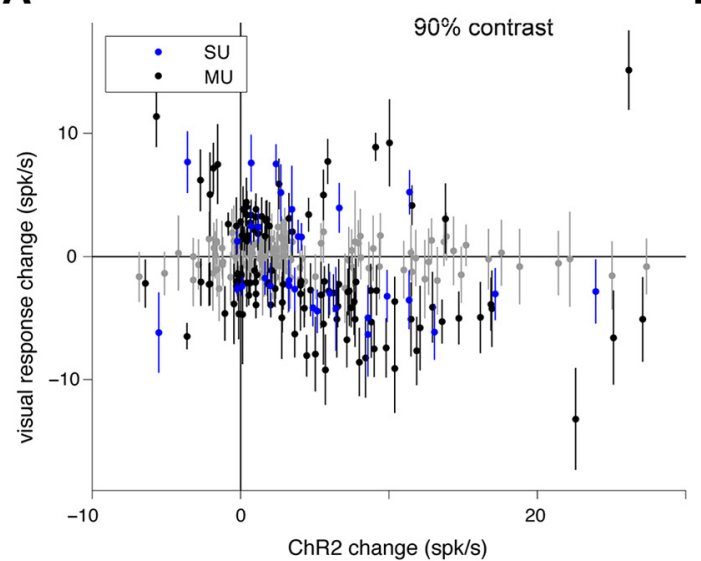

B Visual only
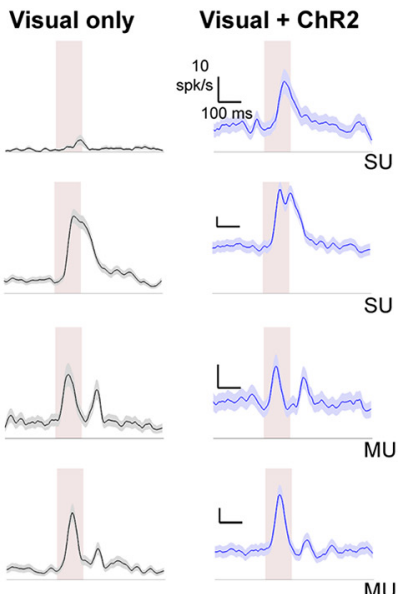

Figure 2. Different units can be sub- or supralinear, although mean of population is near-linear. $\boldsymbol{A}$, Unit responses to excitatory neuron optogenetic (Emx1-ChR2) stimulation, showing that many individual units are significantly supra- or sublinear. $X$-axis, average firing rate change with ChR2 stimulus; $y$-axis, difference between visual responses (90\% contrast; each visual response measured from preceding baseline) with and without optogenetic stimulus. Error bars are SEM. Points that are at least 1 SEM away from horizontal line at zero (linear response) are colored blue (single units; SU) or black (multiunits; MU). Points within 1 SEM of linear are gray. Data are as in Fig. $1 E$ for $90 \%$ contrast, here with SEM for each point, and adding on the negative $y$-axis the few units that are suppressed by stimulation. $34 \%$ of single units are significantly nonlinear $(17 / 50, p<0.01$, KS test), and $28 \%$ of multiunits are significantly nonlinear (67/239, $p<0.01$, KS test). B, Four example units. Pink region shows visual stimulus presentation time. Shaded regions around mean response are SEM. 
relationship between the size of a cell's ChR2 effect and summation (e.g., cells that responded more to ChR2 would be more supralinear, whereas nonresponsive cells would be sublinear). We saw only a small effect of ChR2 response on scaling (Fig. 1C,E). Second, in model simulations (discussed below), where we can definitively identify model cells' ChR2 input, we saw heterogeneity in single units similar to experiment, but scaling had the same trend whether cells received direct ChR2 input or not (see below). Thus, heterogeneity in neurons' responses hints that neurons' connectivity influences summation-that the population response is shaped by connections between excitatory and inhibitory neurons that dynamically respond to each others' activity (van Vreeswijk and Sompolinsky, 1996; Brunel, 2000). Below, using a spiking network model, we explore how connection variation might cause network summation to vary.

\section{Other experimental work finds sublinear summation in macaque visual cortex}

In contrast to this average linear scaling in mouse primary visual cortex, recent work in the monkey primary visual cortex (Nassi et al., 2015) found that neural responses were at times highly sublinear, and averages across neurons were also sublinear. (Previous work in the tree shrew and mouse also found linearity and sublinearity; Huang et al., 2014; Sato et al., 2014.) The experimental approach used by Nassi et al. (2015) does not seem to differ in important ways from our approach: they expressed ChR2 primarily in excitatory neurons (using a CaMKII- $\alpha$ promoter strategy), stimulated an area of the cortex a few hundred micrometers in diameter, and paired $\mathrm{ChR} 2$ and visual stimulation. Because the different results may stem from differences in cortical architecture across species, rather than differences in experimental methods, we sought to determine whether there were features of local cortical circuits that could change response scaling from linear to sublinear.

\section{Model network simulations identify circuit properties controlling input summation}

Because it is difficult to manipulate neural connectivity in vivo, we used numerical simulations of conductancebased model neurons to understand how network connectivity might change response scaling. We constructed networks of 10,000 conductance-based leaky integrateand-fire neurons, 8000 excitatory (E) and 2000 inhibitory (I). We chose realistic parameters for the model neurons, including sparse connectivity (initially $2 \%$ ), and moderate synaptic strengths such that a few tens of excitatory PSPs (EPSPs) were required to push a neuron over threshold. (We explore a range of values of sparsity and synaptic strength below.) These sparse, randomly connected networks produce irregular and asynchronous spontaneous activity (Fig. $3 A$ ) similar to that observed experimentally (Steriade et al., 2001; Destexhe et al., 2003) and show stable responses to external inputs (Vogels and Abbott, 2005). For all simulations, we set the spontaneous average rate of the network to $5 \mathrm{spk} / \mathrm{s}$. There are a variety of single-cell properties that could set neurons' spontaneous rate, but we changed the spontaneous rate by sup- plying a small, constant amount of excitatory input that does not vary with network activity or input, to either excitatory or inhibitory neurons (see Methods).

To determine how different sorts of feedforward inputs affect neurons' responses, we simulated external inputs to $E$ and I cells using two input groups of Poisson spike trains whose rates could be varied independently. As expected, when we varied the external input rates, increasing input to $E$ cells ( $x$ axis) monotonically increased the average network response (Fig. 3B, contour lines; average of all excitatory cells in the network, a measure similar to that obtained by multi-electrode recordings), and increasing input to we cells ( $y$ axis) monotonically decreased the average network response. However, we could hold the average response constant by adjusting the two feedforward inputs. When the average response was constant (along contour lines in Fig. 3B), we still observed changes in response scaling, and those changes depended on the amount of I input.

To assess response scaling in the model (Fig. 3), I began with a combination of $E$ and $I$ input that produced a $15 \mathrm{spk} / \mathrm{s}$ response (chosen because we measured experimentally an average response that peaked near 15 spk/s; Fig. 1C,D). Then, we multiplied both input rates by a single constant and measured the size of the response to the scaled input. We found that when feedforward I input is small, responses are near-linear (Fig. $3 C$ ). This is not surprising, as previous theoretical work using strong local synaptic coupling in models with binary (van Vreeswijk and Sompolinsky, 1996) or current-based (Brunel, 2000) neurons showed that networks can produce linear responses although individual neurons in cortical networks are nonlinear (Priebe and Ferster, 2008). However, these models did not characterize the effects of varying feedforward $E$ and I input separately, and so we varied feedforward I input in the conductance-based model. Indeed, when feedforward I input was varied, we observed deviations from linearity. Although the spontaneous spike rate and the spike rate response to a single stimulus alone were both held constant with and without feedforward inhibition, increasing stimulus strength showed more sublinear response scaling when feedforward inhibition was present.

\section{Local connectivity changes summation only in the presence of feedforward inhibition}

Although adding feedforward inhibition induced some sublinearity, we wished to know whether more dramatic nonlinearities were possible. Therefore, we next (Fig. 4) changed local recurrent connectivity between and among $E$ and I populations and measured how those connectivity changes affected response scaling. Fig. 4 shows the effects of varying two local connections (first, strength of synapses from E to I, and second, strength of synapses from I to I) to illustrate the range of effects I observed. To implement varying connectivity in the model, we added additional connections between two neuronal populations (e.g., E to I or I to I) with the same sparsity as the network. I then varied the strength of those additional connections and measured effects on response scaling. 
A

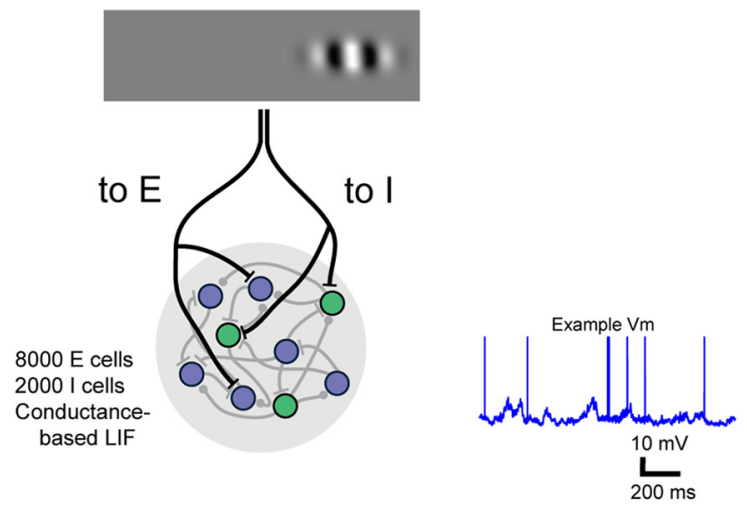

B

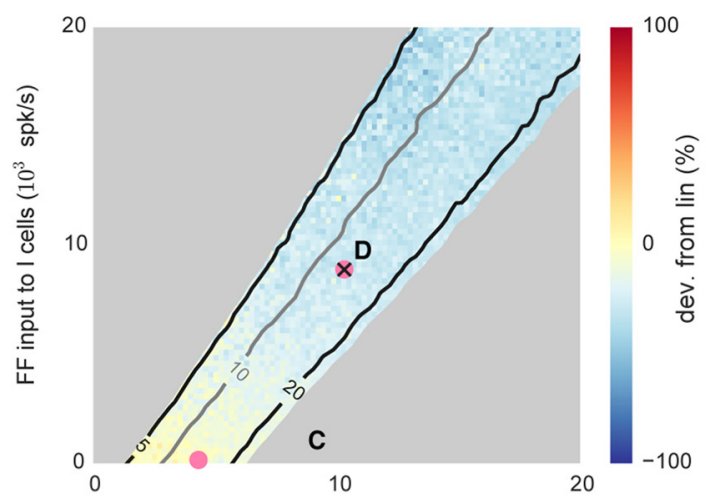

FF input to $\mathrm{E}$ cells $\left(10^{3} \mathrm{spk} / \mathrm{s}\right)$
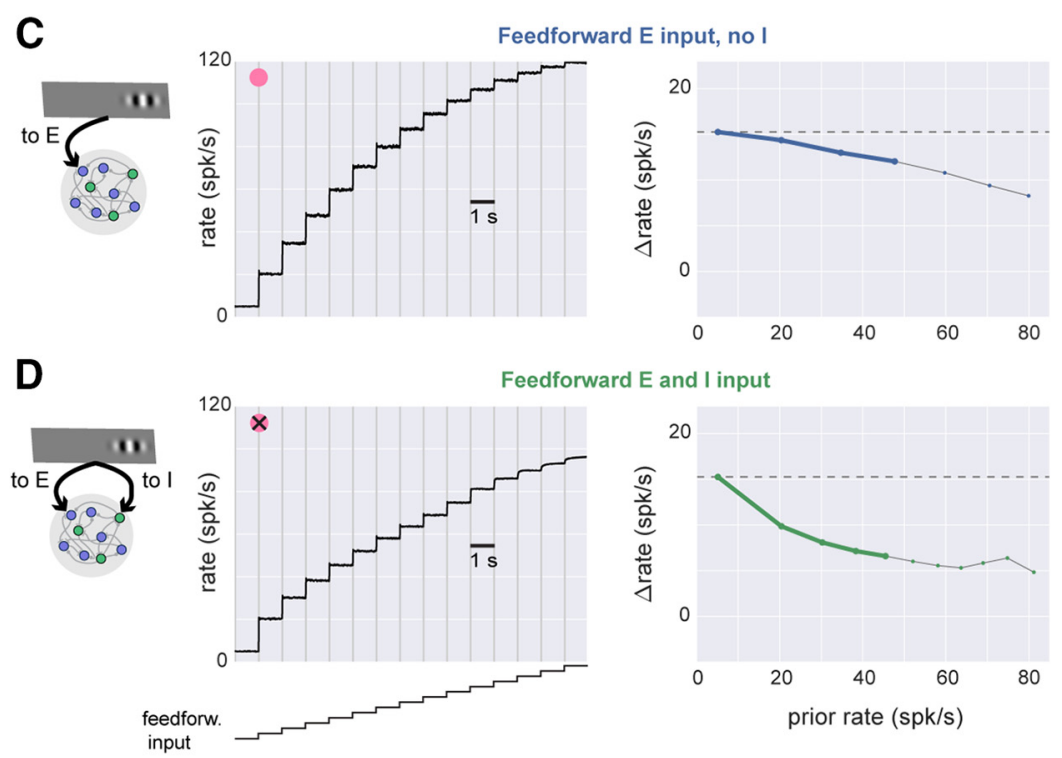

Figure 3. Spiking model shows sublinear scaling with feedforward inhibition. $\boldsymbol{A}$, Schematic of network architecture. Blue, E cells; green, I cells. The conductance-based spiking model produces stochastic $V_{m}$ and spikes as seen in vivo, and an example membrane potential $\left(V_{m}\right)$ trace from one excitatory cell is shown. $\boldsymbol{B}$, Response scaling as feedforward (FF) input to $\mathrm{E}$ and I cells is varied. To measure response scaling, inputs to $\mathrm{E}$ and/or I cells with rate given by $x$ and $y$ axes are delivered, and average response over all $E$ cells is measured. Then, the $E$ and I input rates are multiplied by a constant (here, 2), and the size of the second response is compared to the first. Percentage change shown by color: yellow, second response is similar (linear); blue, second response is smaller (sublinear). Contour lines show first response (spk/s). Response rates $<5$ and $>20 \mathrm{spk} / \mathrm{s}$ are masked (gray). Average spontaneous rate is adjusted to $5 \mathrm{spk} / \mathrm{s}$ (Methods), and 33\% of network neurons receive external input, to approximate the sparse set of cortical neurons that typically respond to sensory inputs (Fig. 1). There is a gradual increase in sublinearity moving up and to the right in the figure. Pink points show $\mathrm{E}$ and I rate combinations used in $\boldsymbol{C}$ and $\boldsymbol{D}$. $\boldsymbol{C}$, Near-linear responses to a range of input sizes when feedforward input is provided to E cells only. Parameters here are indicated by pink dot in $\boldsymbol{B}$, and first two responses here are the same two responses used to compute percentage change shown in color there. Left, average rates; right, same data replotted showing 


\section{continued}

change (spk/s) in response ( $y$-axis) as a function of prior response ( $x$-axis). For these plots, a linear response is a horizontal line (dashed gray line). Heavy lines, prior rates $<50 \mathrm{spk} / \mathrm{s}$, highlighting for visual clarity rates far from potential saturation caused by absolute refractory period $(3 \mathrm{~ms})$. $\boldsymbol{D}$, Sublinear responses to a range of input sizes when input provided to both $\mathrm{E}$ and I cells. Same conventions as $\boldsymbol{C}$. In this case, heavy green line in right panel lies farther below horizontal than heavy blue line in $\boldsymbol{C}$, showing more sublinear scaling.

With only feedforward input to E cells (Fig. 4A,C,E), we found that changing network connections did not dramatically affect response scaling. Changing the connectivity could change the gain of the network (the size of the response to a constant input; Fig. $4 A$, contour lines), but response scaling was nearly linear (Fig. $4 A$, plot is yellow throughout; Fig. 4C,D: black lines lie close to horizontal dotted line). At high firing rates, we consistently saw
A

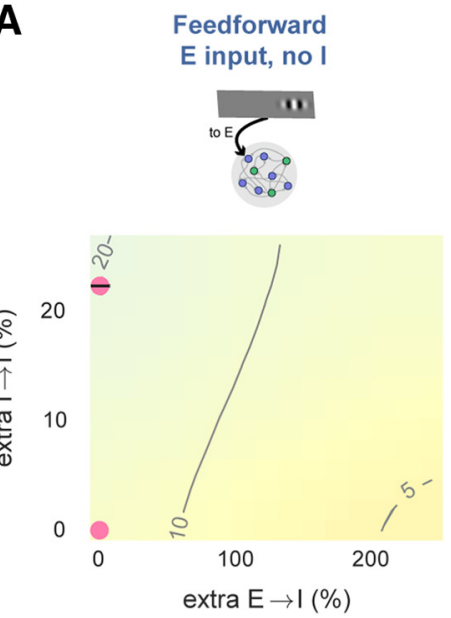

C

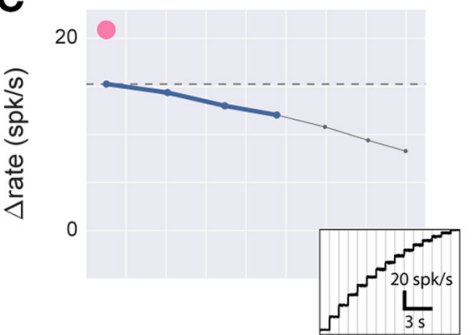

E

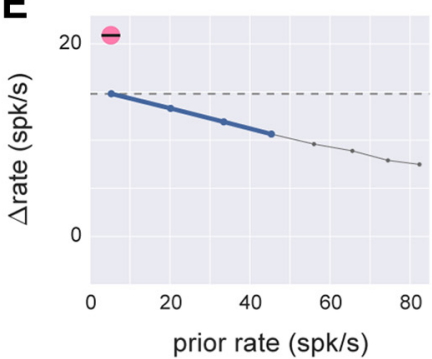

B Feedforward

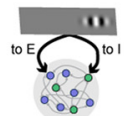

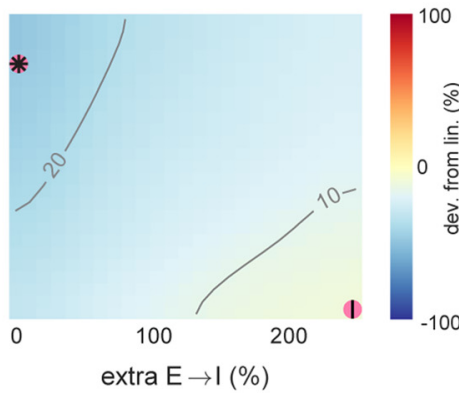

$\mathrm{D}$

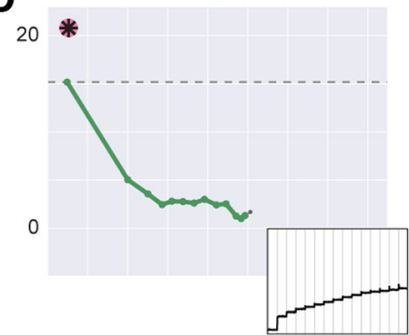

$\mathbf{F}$

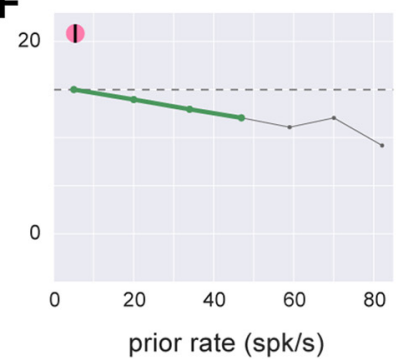

Figure 4. With feedforward inhibition, network model can produce linear or sublinear responses. $\boldsymbol{A}$, Simulations with feedforward input to E cells only, while local network connectivity is varied. $x$-axis, E-to-I connection strength; $y$-axis, I-to-I connection strength. Axes give percentage change in total synaptic input that a single cell receives from one (E or I) population (see Methods), where zero is a balanced network (e.g. Fig. 3) with equal probability of synapses onto $E$ and I cells. Other conventions as in Fig. $3 B$ (contour lines show evoked response to first stimulus, color shows percentage difference in response to doubled external stimulus). Spontaneous rate and external stimulus rates are constant for entire panel. $\boldsymbol{B}$, Simulations with feedforward input to $\mathrm{E}$ and I cells while local connectivity is varied. Pink symbols show parameter regions where scaling is sublinear (stronger $|\rightarrow|$ connectivity) or linear (stronger $\mathrm{E} \rightarrow$ l connectivity). $\boldsymbol{C}$, Scaling plot (response size as a function of previous rate) for parameters shown by pink dot in $\boldsymbol{A}:$ no extra local connections, feedforward E only, same parameters as Fig. 3C. Inset, time course of responses to the step stimulus; subtracting each rate from rate at the previous step gives $y$-axis in main panel. $\boldsymbol{D}-\boldsymbol{F}$, same plots, using parameters shown by corresponding pink dots in $\boldsymbol{B}$. Comparing $\boldsymbol{D}$ and $\boldsymbol{E}$ shows that large sublinearity can be produced by extra $I \rightarrow \mid$ connections only with feedforward inhibition. Comparing $\boldsymbol{D}$ and $\boldsymbol{F}$ shows that linearity can also be achieved with feedforward inhibition if $\mathrm{E} \rightarrow \mathrm{I}$ connectivity is strengthened. 
moderate increases in sublinearity, which seems likely to be due to effects of the 3-ms absolute refractory period. (To focus on rates well below the refractory period, I show rates above $50 \mathrm{spk} / \mathrm{s}$ as light gray lines in Fig. 4C,D.) I also varied all pairwise combinations of E-to-I connectivity, as well as feedforward E-and-I input strength, and found that without feedforward inhibition, responses never showed substantial nonlinearity. Thus, the linear scaling we had observed in the model when delivering input to $\mathrm{E}$ cells only was robust to changes in local connectivity. In sum, without feedforward inhibition, scaling was approximately linear, and local connectivity changes had little effect.

Near-linear scaling was consistently seen when feedforward input arrived to E cells, but when feedforward input arrived to both $E$ and I cells, responses could be either linear or sublinear. When we increased local I-to-I connection strength (Fig. 4B, y axis), sublinearity was observed (Fig. $4 D$; plot parameters correspond to pink asterisk in Fig. $4 B$, in blue region of plot). But increased E-to-I connection strength (Fig. $4 B, x$ axis) led to increasingly linear scaling (Fig. 4E; plot parameters correspond to pink I symbol in Fig. 4B). The sublinear scaling produced by stronger I-to-I connectivity was dramatic. As with all the time course plots (Fig. 4C-F), I chose input strength so that the first firing rate response was $15 \mathrm{spk} / \mathrm{s}$, but when I-to-I connectivity was increased, subsequent firing rate responses fell as low as $1 \mathrm{spk} / \mathrm{s}$ (Fig. 4D).

It appears that increased I-I coupling creates sublinearity for the later (stronger) stimuli by changing the scaling of the $E$ cells in the network. Analytical work shows it is possible for the $E$ cells to show a supralinearity for small inputs that becomes more sublinear at larger inputs (Ahmadian et al., 2013; Sanzeni et al., 2017). Stronger I-I connections reduce amplification of inputs to I cells, but at the same time increase amplification for inputs to $E$ cells. This increase in amplification of $E$ responses can increase the range of input that gives supralinearity in $\mathrm{E}$ cells. It is consistent with the responses we saw in simulation (Fig. $4 C, D$, insets) that, after changing I-I connections, the first response lies on the initial supralinearity, making the rest of the responses more sublinear. (In all the panels of Fig. 4, the I-cell responses show no large nonlinearities, suggesting that it is not changes in I-cell responses that drive the sublinearity we saw.) Whether I-I connections can change very much in vivo is unclear, but anatomic studies suggest that some changes can occur, as inhibitory cells modify their dendritic structure over time (Chen et al., 2011).

In sum, the numerical simulations show that local connectivity changes can dramatically affect response scaling, but only in the presence of feedforward I input.

\section{Connectivity effects on summation do not depend on connection sparsity or strength}

We next examined whether synaptic strength and connection sparsity can change the role of feedforward inhibition in response scaling. We expected that varying the total recurrent input that neurons receive would change nonlinearity of responses (as predicted by theory, van Vreeswijk and Sompolinsky, 1996; Ahmadian et al., 2013), as long as the network remained stable. Therefore, we varied total input in two ways, by varying connection sparsity and synaptic strength (Fig. 5). Experimental estimates of local connection sparsity range as high as $10 \%-$ $20 \%$ (i.e., each neuron connects to $10 \%-20 \%$ of nearby neurons; Braitenberg and Schüz, 2001; Lefort et al., 2009). But the effective sparsity of connections might be lower, as connection probability in cortical networks is known to fall off with distance. Average network connection probabilities might thus be lower than the measurements obtained for nearby neurons. Therefore, to examine the effects of changing connection probability, we varied sparsity between $2 \%$ and $20 \%$. We found that in all these cases, adding feedforward inhibitory drive allowed more sublinear responses (Fig. 5; green lines always lie below blue lines in Fig. 5A). We observed more linear scaling when we increased the strength of all synapses together and a bigger range of possible scaling (from supralinear to sublinear) when we decreased synaptic strength. These results show that, in networks that use a range of connection strength and sparsity, feedforward inhibition enables local $E$ and I connectivity to have similar effects on response scaling, although the networks became more linear as connectivity strength increased.

\section{Summation here and in past data can be explained by a model with feedforward inhibition}

Next, we asked whether a model that incorporates realistic optogenetic input shows the same scaling dependence on feedforward inhibition we observed. Up to this point, I had examined the behavior of simulated networks only by scaling a feedforward (spiking) input (Figs. 3-5). I implemented this feedforward input to simulate the way input spikes change conductance in neurons, by modulating the firing rate of a (Poisson) stochastic point process. Using these input spike trains, the sum of feedforward synaptic inputs in a given network neuron has substantial fluctuations about its mean. In contrast, experimental ChR2 stimulation activates many channels and produces conductance changes with much smaller fluctuation about the mean. Thus, it might be possible that the scaling behavior we studied experimentally, with ChR2 combined with visual stimuli, would differ from the combinations of feedforward input simulated in Figs. 3-5. To determine whether there was a difference, we simulated ChR2 input by changing conductance and combined this with feedforward input (Fig. 6). I found that combinations of ChR2 and visual inputs produced effects qualitatively similar to those I had previously seen. Combinations of simulated ChR2 and visual input (Fig. 6A) showed slightly increased sublinearity compared with a single scaled visual input (cf. Fig. 3B). (I also saw some slight sublinearity in my measurements of responses to combined ChR2 and visual input in mouse V1, Fig. 1.) However, as with simulated visual input (Figs. 3-5), we found that with paired conductance (ChR2) and spiking (visual) inputs, more sublinearity is possible when the feedforward input combines inhibitory and excitatory targets than when feedforward input targets only excitatory neurons (Fig. 6B,C). Also, in the presence of feedforward inhibition, moderate 
A

$10 \%$ sparsity, stronger local connections
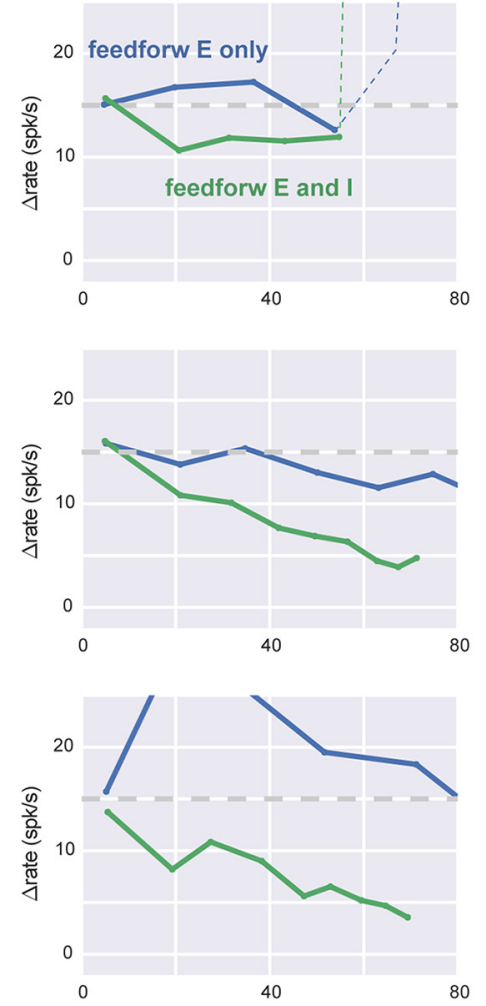

$10 \%$ sparsity weaker local connections

$2 \%$ sparsity
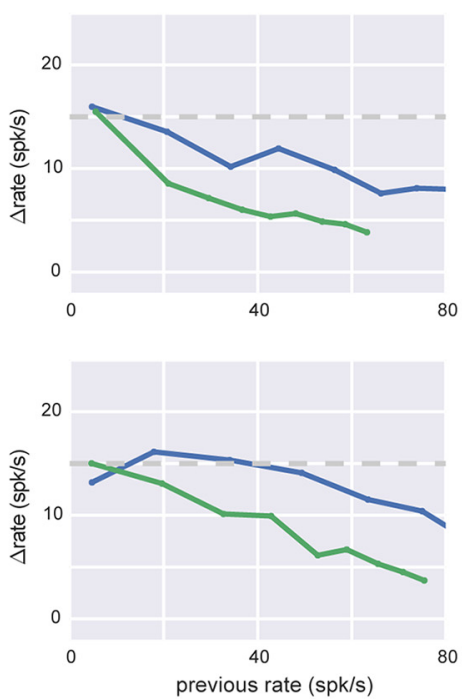
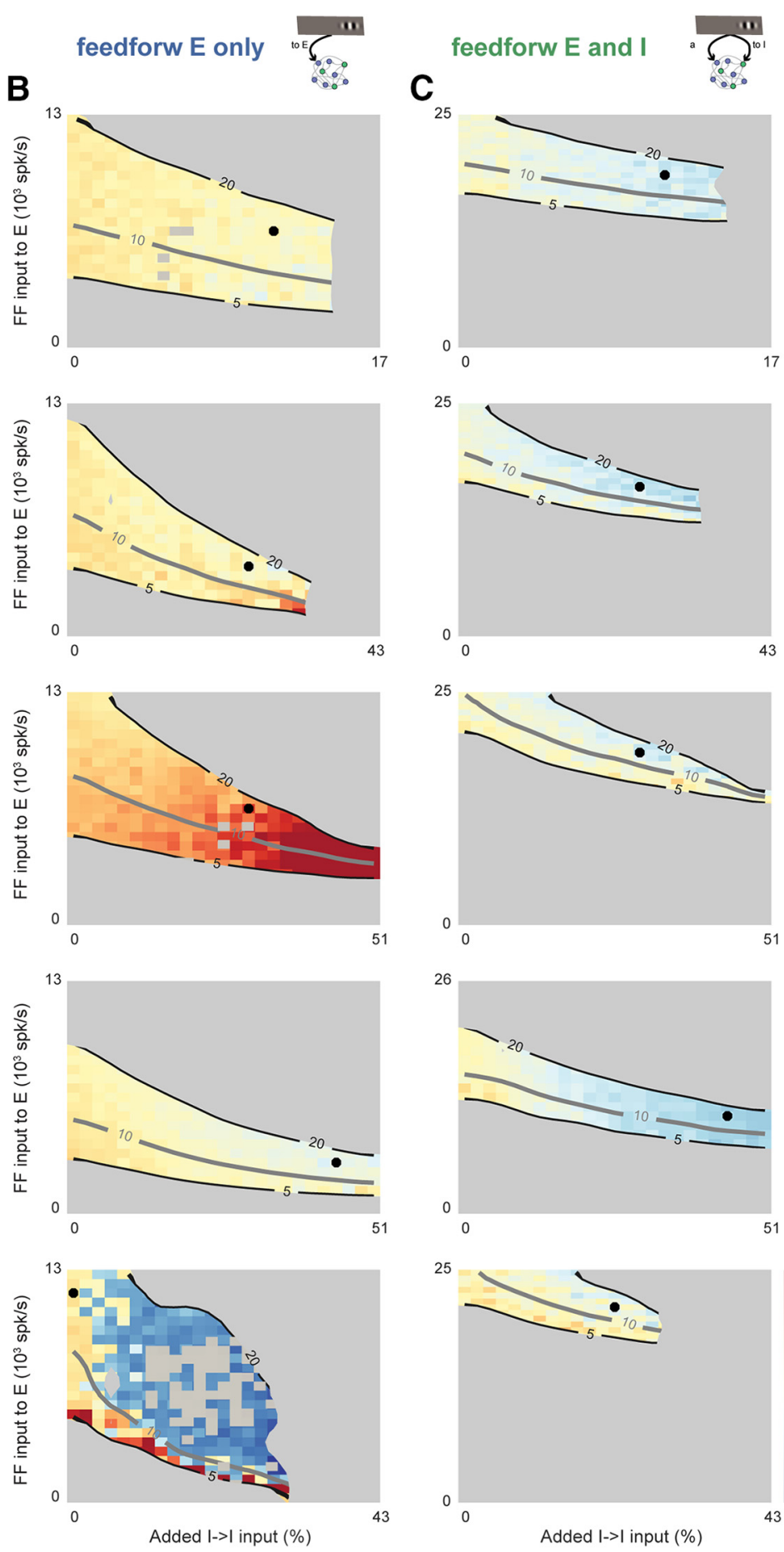

Figure 5. Feedforward inhibition leads to sublinearity in networks with a range of recurrent synaptic sparsities and synaptic strengths. Top row, simulations in the conductance-based network with $10 \%$ connectivity, with strong synapses (each cell receives $10 \times$ more $\mathrm{E}$ and I input than in the networks of Figs. 3 and 4). Other rows show networks with different sparsity and synaptic strength. The network of Figs. 3 and 4 is the fourth row ( $2 \%$ sparsity, $1 \times$ strength). $\boldsymbol{A}$, Scaling plots showing network response as a function of prior rate before stimulus. Blue, feedforward $\mathrm{E}$ input only, parameters shown in column B; green, feedforward E and I input, corresponding parameters shown in column C. In all rows, feedforward inhibition (green) allows more sublinearity than feedforward excitation alone (blue). Dashed line, top row: network instability (rates diverge). $\boldsymbol{B}$, Average network response as I-I synaptic strength ( $x$-axis) and feedforward $E$ input ( $y$-axis) are varied. No feedforward inhibition. Black dot shows parameters used to plot blue line in $\boldsymbol{A}$ (parameters chosen to maximize sublinearity). Gray regions mask areas where evoked rates are $<5$ or $>20 \mathrm{spk} / \mathrm{s}$, or where network was unstable (rates diverged to maximum rate given by refractory period). Other conventions as in Figs. $3 B$ and $4 A, B$. $\boldsymbol{C}$, network response as a function of $\mathrm{I} \rightarrow \mathrm{l}$ and feedforward $\mathrm{E}$ input, in the presence of feedforward inhibition. Individual gray squares seen in fifth row $(20 \%$ sparsity) column B, inside the 5-20 spk/s contours indicate strongly irregular (nonmonotonic) response scaling: strong sublinearity for 
continued

at least one stimulus step, when both previous and later responses were linear or supralinear. Feedforward inhibition arrival rate to stimulated cells for each row, from top: $14 \mathrm{k}, 14 \mathrm{k}, 19 \mathrm{k}, 11 \mathrm{k}, 17 \mathrm{k} \mathrm{spk} / \mathrm{s}$, chosen to give a $15 \mathrm{spk} / \mathrm{s}$ response for $3 \times$ the feedforward excitatory rate that alone produces a $15 \mathrm{spk} / \mathrm{s}$ response (see Fig. $3 B$ ). Fourth row (2\% sparsity, same network as Figs. 3 and 4$)$ uses $40 \%$ extra $\mathrm{I} \rightarrow \mathrm{E}$ connections to show that linear responses are robust to many forms of connectivity variation.

changes in network connectivity can modify scaling behavior (Fig. 6D). In sum, in the models that simulate visual input alone (Figs. 3-5) and the models that simulate combined visual and ChR2 (conductance) inputs (Fig. 6), the role of feedforward inhibition and I-I connectivity in response scaling is similar.

We next asked what combinations of connectivity and feedforward input could describe both my data and past measurements. We constructed a model with combined visual (spiking) and ChR2 (conductance) inputs and fitted evoked rates to my data. My data (Fig. 6E) were wellmatched by the simulations that showed small sublinearity (Fig. $6 A-D$ ). The data were similar to two different sets of network simulation parameters. It was possible for networks with only feedforward excitation to show responses describing the data (Fig. 6B), but networks with

\section{Simulations}

A

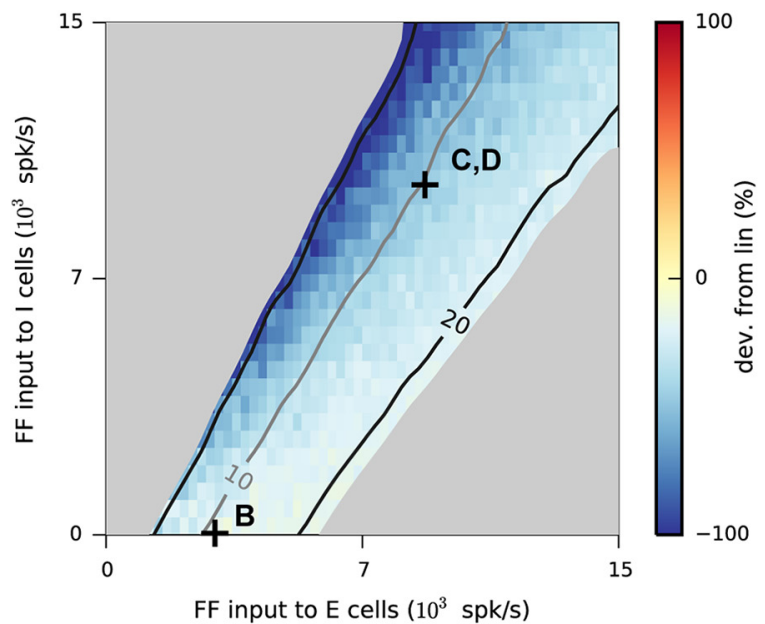

\section{E Data}

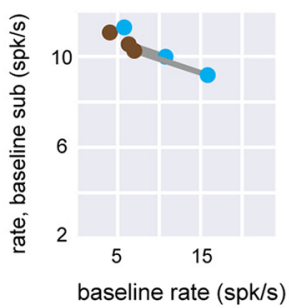

\section{B}
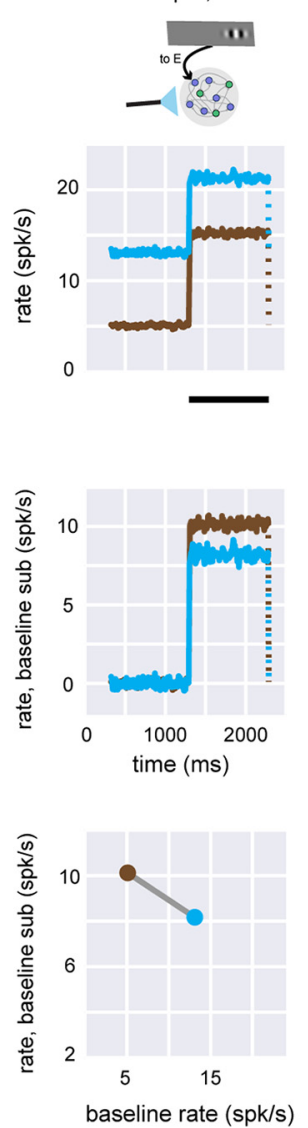
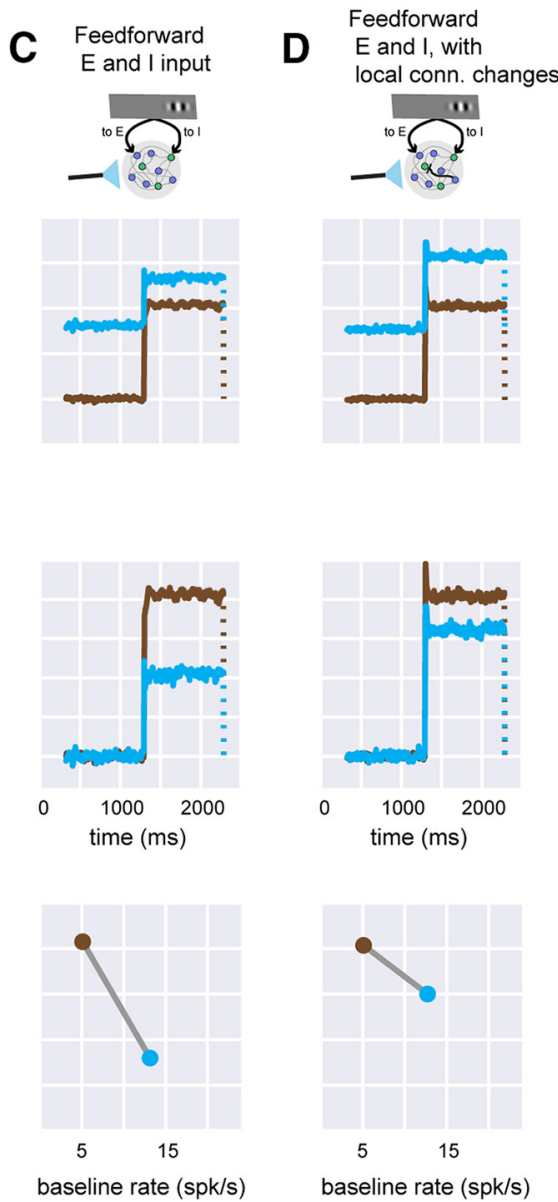

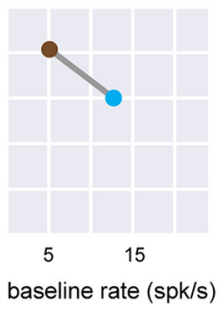

Figure 6. Experimental linear scaling can be replicated in networks receiving feedforward inhibition. $\boldsymbol{A}$, Simulation where conductance steps (ChR2 input) and feedforward Poisson trains (visual input) are combined. Strengths of feedforward $E$ input ( $x$-axis) and feedforward I input ( $y$-axis) are varied while spontaneous rate is set to $5 \mathrm{spk} / \mathrm{s}$. Connection sparsity is $2 \%$. Other conventions as in Fig. 3B. Symbols (+) show values of E, I input used in $\boldsymbol{B}-\boldsymbol{D}$. $\boldsymbol{B}$, Network responses when feedforward input is supplied to $E$ cells only. Top row, network responses (mean of E cell rates). Brown, feedforward Poisson (visual) input only; cyan, conductance (ChR2) input combined with visual input. Conductance increase lasts for the full duration of the cyan trace. Visual input duration is shown by black bar (bottom of plot). Dotted line indicates rates return to previous baseline when feedforward input ends. Second row, same data as top row, with baseline rate subtracted. Third row, response ( $y$-axis) as a function of rate before feedforward input begins ( $x$-axis). $\boldsymbol{C}$, Same network simulations with feedforward input to both $\mathrm{E}$ and I cells (parameters marked by $\mathrm{C}$ in $\boldsymbol{A}$ ). $\boldsymbol{D}$, network receiving feedforward input to both $\mathrm{E}$ and I cells, but with stronger local connections from E to I cells (compare Fig. 4, with similar effect for two feedforward Poisson inputs instead of feedforward input paired with conductance step as shown here). $\boldsymbol{E}$, data from Fig. $1 C$ plotted to show how responses scale as baseline is changed. Three lines (brown, no ChR2; cyan, with ChR2) are the three groups of recorded neurons shown in Fig. $1 C$. 


\section{PV-ChR2: $\quad$ Data}

A
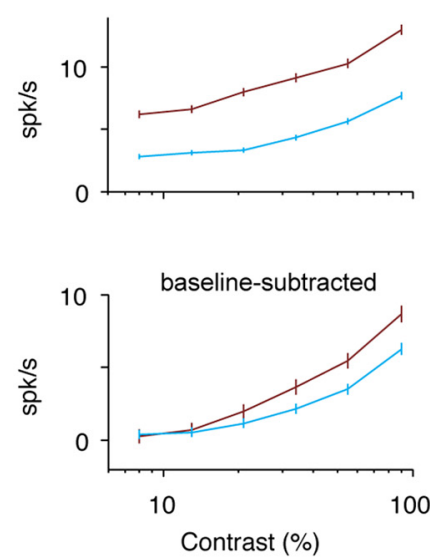

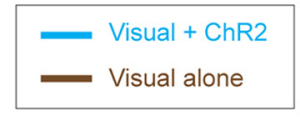

B

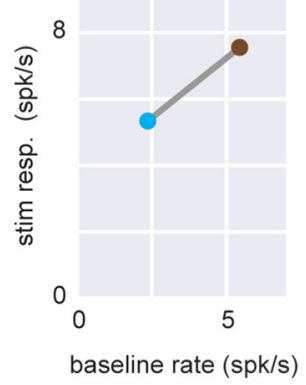

Simulations

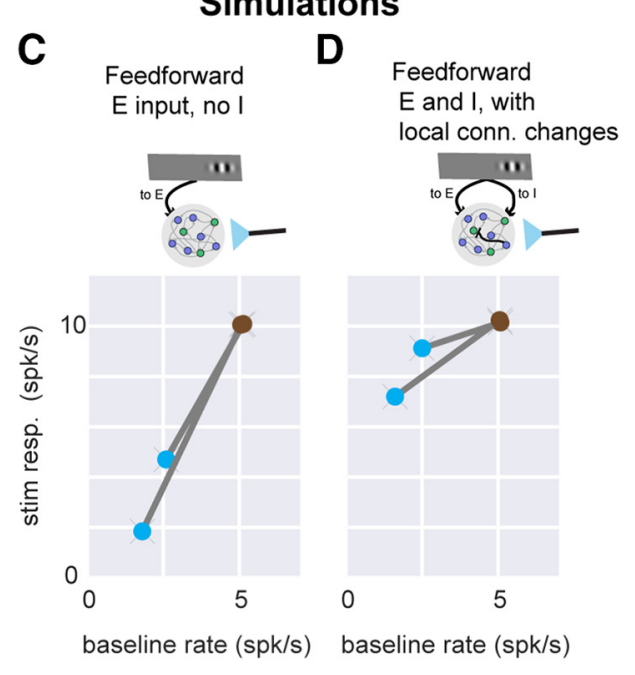

Figure 7. PV-ChR2 stimulation data support the recurrent model with feedforward inhibition. A, Moderately sublinear scaling of visual responses is seen when PV neurons are optogenetically stimulated. (Data set previously reported in (Glickfeld et al., 2013)). Same conventions as in Fig. 1D. $n=43$ units, $6 \mathrm{SU}, 37 \mathrm{MU}$. B. Response sizes plotted as a function of baseline rate; same conventions as bottom panels in Fig. $6 B-D$. Stimulation of PV inhibitory neurons lowers baseline firing rates (here $2.3 \times$ reduction), so visual + ChR2 response (blue point) is to the left of visual only (brown). $\boldsymbol{C}, \boldsymbol{D}$, Model (with feedforward inhibition) that best fits $\mathrm{E}$ neuron stimulation data also describes moderate sublinearity seen in PV-ChR2 stimulation. $\boldsymbol{C}$, Model with feedforward input to E cells only (same model as in Fig. $6 B$ ) shows very strong sublinearity. Two lines show two different strengths of optogenetic input to I cells (chosen to produce $2 \times$ or $3 \times$ decrease in baseline rates). $\boldsymbol{D}$, Model with feedforward input to $\mathrm{E}$ and I cells and stronger local E-to-I connectivity (same model as in Fig. $6 D$ ), shows a range of sublinear scaling similar to that seen in the experimental data $(\boldsymbol{A}, \boldsymbol{B})$.

both feedforward excitation and inhibition could also describe the data when the network local connectivity was adjusted (Fig. 6D). Because feedforward inhibition is a common feature of cortical networks in many species (Douglas and Martin, 2004), a model using feedforward inhibition seems a good choice to describe experimentally measured response scaling. Moreover, as described above, with feedforward inhibition, changes in local (e.g., I-I) connectivity can change response scaling from linear to sublinear, describing not just this data but also past data. These simulations show that a wide regime of cortical scaling behavior, from linear (as seen here in mouse V1 and also in the tree shrew; Huang et al., 2014), to strongly sublinear (as seen in primate V1; Nassi et al., 2015), can be achieved by a model with feedforward inhibition. In sum, the simulations show that a model with feedforward inhibition can describe both my data and past observations.

With this model, we could compare response scaling of neurons that received direct ChR2 input (33\% of model E neurons, see Methods) to those that received no ChR2 input and thus had optogenetic responses due only to recurrent input from other cells in the network $(67 \%$ of $E$ neurons). Using a model network in which the average across all E neurons was sublinear (with feedforward inhibition but no local connection changes; Fig. 6C), I found that both directly driven and recurrent-only neurons show sublinear scaling (all E cells, mean $-0.7 \mathrm{spk} / \mathrm{s}$ change in visual stimulation response per $1 \mathrm{spk} / \mathrm{s}$ change in baseline rate; directly driven E cells, $-0.6 \mathrm{spk} / \mathrm{s}$ per unit baseline; non-directly driven E cells, $-0.9 \mathrm{spk} / \mathrm{s}$ per unit baseline), though the directly driven cells, as expected, showed larger ChR2 responses (all E cells, $7.9 \pm 15$ spk/s; directly driven, $22.7 \pm 20 \mathrm{spk} / \mathrm{s}$; non-directly driven, $3.0 \pm 8.9 \mathrm{spk} / \mathrm{s}$, mean $\pm \mathrm{SD}$ ). Thus, though experimentally it is difficult to reliably distinguish neurons that receive direct ChR2 input from those that do not, both populations show similar scaling in the model, supporting analyses of scaling that consider both populations without definitively separating them. Also, in the model, the larger the ChR2 firing rate change, the larger the change in visual response (in spikes per second). This is consistent with the data in Figs. 1 and 2: the average scaling in the data is slightly sublinear, and the neurons with the largest ChR2 effects show slightly increased sublinearity.

\section{PV neuron stimulation effects are explained by the model with feedforward inhibition}

We next tested the model against data obtained by pairing visual and optogenetic stimulation of parvalbuminpositive (PV) cells. A majority of cortical PV inhibitory neurons are soma-targeting fast-spiking basket cells (Kawaguchi and Kubota, 1997; Tremblay et al., 2016), which are well positioned to act as the balancing population in the network models. We found that stimulating PV neurons with ChR2 in awake mice produces a moderate suppression of visual responses, with a larger change in baseline rates than in stimulus responses. As before, we measured the visual response relative to the preceding baseline firing rate, which is changed by optogenetic stimulation. The optogenetic stimulation lowered 
the baseline firing rate by a substantial amount (from 5.4 to $2.4 \mathrm{spk} / \mathrm{s}$, a $57 \%$ reduction; $p<10^{-7}$, Wilcoxon test) and reduced the response to a high-contrast visual stimulus by a smaller amount (Fig. 7A,B; from 7.6 to $5.3 \mathrm{spk} / \mathrm{s}$ or 29\%; $p<10^{-6}$, Wilcoxon test). The additive baseline effect and smaller sublinear effect on responses we observed is consistent with prior studies activating PV neurons in mouse visual cortex (but see Atallah et al., 2012; Lee et al., 2012; Wilson et al., 2012), where despite some differences in sharpening of orientation tuning (perhaps explained by threshold-linear models Atallah et al., 2012, 2014; Phillips and Hasenstaub, 2016), additive effects with some sublinearity were seen.

We then used this PV-ChR2 stimulation data to determine which models in Fig. 6 fitted both the excitatory and PV stimulation mouse V1 data. As with the simulations in which excitatory neurons received ChR2 (conductance) input, we simulated the effects of optogenetic stimulation of PV cells by delivering a conductance input to PV neurons in the models. We adjusted the size of the conductance input to match the firing rate changes in the data (Fig. 7C). The two models that fitted the near-linear responses to excitatory stimulation (Fig. 6) are the model without feedforward inhibition (Fig. 6B) and the model with feedforward inhibition and local synapses adjusted to produce near-linear responses (Fig. 6D). For each of those two models, we simulated optogenetic input to PV cells and measured the change in visual response size with and without optogenetic PV input. We found that the model without feedforward inhibition disagreed with the PVChR2 data, displaying very strong suppression (Fig. 7C). Only the model with feedforward inhibition (Fig. 7D) showed the same scaling (moderate suppression) in the PV-ChR2 data. The reduced suppression in the model with feedforward inhibition might be due to a smaller proportion of $\mathrm{PV}$ total input coming from optogenetic stimulation in that model, compared with the model in which PV cells receive no direct feedforward input. Optogenetic perturbations of excitatory and PV-positive cells are described by a cortical recurrent network model that requires feedforward inhibition.

In sum, my data show that average response summation for excitatory input in mouse V1 is close to linear, although individual cells can be nonlinear. Linear summation holds even for substantial shifts in firing rate (ChR2induced firing rate changes of 10-15 spk/s, approximately the same size as the maximum visual response; Fig. 1). Using a numerical model of conductance-based spiking neurons, we found that response scaling is affected dramatically by synaptic connectivity. Moderate changes in synaptic coupling ( 20\%) between inhibitory cells can change response scaling from linear to sublinear (Figs. 4-6). Further, the change in inhibitory-to-inhibitory (I-I) connectivity that leads to sublinear summation only yields such sublinear summation in the presence of feedforward inhibition.

\section{Discussion}

It might seem surprising that we experimentally observed linear responses and not purely divisive normal- ization, where adding an additional stimulus yields reduction of the responses to a single stimulus. This form of sublinear summation has been observed in different visual cortical areas of several species. Linear summation, on the other hand, is also commonly seen at various stages of sensory systems, and both linear and sublinear responses may be useful at different levels (Carandini and Heeger, 2012). Linear summation may be more desirable when responses at different locations should receive equal weight, as when an organism must sensitively detect a distant predator, or when spikes that occur at different times should produce the same downstream effect. In fact, computer vision systems often use both linear and normalization steps in distinct layers or networks (Carandini and Heeger, 2012; Yamins and DiCarlo, 2016). Experimentally, normalization is usually measured with sensory stimuli, not with direct cortical input, and thus normalization might partially depend on subcortical (e.g., thalamic gain control; Bonin et al., 2006) or feedback effects. Whether responses are linear or sublinear (divisive) might also be controlled by network activity level (Phillips and Hasenstaub, 2016), or, as studied here, by intracortical connectivity in $\mathrm{E}$ and I populations.

The linear responses we observed with excitatory optogenetic stimulation in mouse primary visual cortex are similar to those seen in tree shrew visual cortex (Huang et al., 2014) but different from the sublinear responses seen in macaque visual cortex (Nassi et al., 2015). Although variation in evoked firing rates may be an explanation for those differences, our simulations show that a broadly similar cortical architecture can support both kinds of scaling, subject to moderate adjustments in local connectivity. The linear responses we saw in the mouse differ from those of Sato et al. (2014), who also delivered combinations of excitatory optogenetic and visual input to mouse V1 neurons and found sublinearity under certain conditions. However, Sato et al. used an experimental approach different from the other three studies (macaque, tree shrew, and the present study in mouse), in which they optogenetically elicited antidromic input spikes by stimulating the contralateral hemisphere from which they were recording. Comparing these two types of input may shed additional light on how cortical circuits transform inputs to outputs.

To stimulate many V1 neurons, I delivered optogenetic input to multiple neurons simultaneously. I used a blue light spot a few hundred micrometers in diameter, comparable to the region of mouse V1 activated by my small visual stimulus. Many neurons in the cortex change their firing rates in response to even small sensory stimuli (Bonin et al., 2011; Van Essen et al., 1984). Anatomically, sensory input that arrives to multiple cells is common, as in the case of divergent feedforward thalamic input to the cortex (Reid, 2001). Single axons from the thalamus often ramify across several hundred micrometers of the cortex (Garraghty and Sur, 1990; Braitenberg and Schüz, 2001), and thalamic axons projecting to the visual cortex can make synapses on dozens of excitatory cortical cells (Freund et al., 1989). 
Optogenetic stimuli may lead to firing rate changes in other parts of the brain besides the area stimulated. But perhaps because the majority of synapses made by cortical neurons are within the same cortical area, local intracortical effects for optogenetic stimuli like these have been observed to be larger than effects on the visual thalamus (Olsen et al., 2012; Li et al., 2013), although the visual thalamus (dorsal lateral geniculate) receives a strong projection from V1 (Reid, 2001). Thus, the neurons best suited to act as the recurrent population in the model may be other V1 neurons, and perhaps even neurons within a few hundred micrometers of the neurons receiving input, where the probability of recurrent connectivity is highest (Lefort et al., 2009). However, other neurons in the brain could also in principle contribute to the recurrent population.

My results show that network mechanisms can contribute to response summation. The model neurons are leaky integrate-and-fire neurons, so individual model neurons sum their subthreshold inputs linearly, and the nonlinear spiking responses we characterized likely arise from how $E$ and I neurons interact. We chose this model architecture because I judged it the simplest model that could capture both excitatory-inhibitory interactions and also single-cell nonlinearities due to refractory period, $V_{m}$ fluctuations, spike threshold, and conductance changes (Chance et al., 2002; Richardson, 2004). There are, however, other single-cell mechanisms, such as short-term synaptic plasticity or dendritic nonlinearity (Häusser et al., 2000; Silver, 2010), that might additionally contribute to even more nonlinear summation, both below threshold and in spike responses. On the other hand, dendritic nonlinearities might also have roles that do not affect scaling; for example, nonlinearities can be used to amplify distant input synapses so that different synapses produce equal responses at the soma (Katz et al., 2009).

We adjusted synaptic coupling between ( $E$ and/or I) populations by changing the strength of a set of fixed connections between the desired populations. Because, in sparse networks like this, neurons share only a small fraction of their input, we expected increases in synaptic strength to achieve the same qualitative result as adding new synapses, even if the two types of changes may not have exactly proportional effects on the behavior of the network. Fig. 5 shows that feedforward inhibition allows more sublinearity across changes in both synaptic strength and synapse number.

Feedforward inhibition is included in the canonical cortical microcircuit framework (Douglas and Martin, 2004) because it is a stereotypical feature of many cortical areas. In sensory cortical areas, including the visual cortex, it has been observed that input thalamic neurons make synapses onto both excitatory principal cells and inhibitory basket cells. Such feedforward inhibitory connectivity has been observed with both anatomic and physiologic methods (Isaacson and Scanziani, 2011). Because inhibitory basket cells project strongly back to excitatory cells, inhibitory changes due to thalamic input arrive to principal cells a few milliseconds after the first excitatory changes. This delay of a few milliseconds be- tween the arrival of excitation and inhibition can be used to align spike outputs of cortical neurons (Swadlow, 2003; Gabernet et al., 2005; Cruikshank et al., 2007; Tiesinga et al., 2008; Tremblay et al., 2016). Beyond shaping the timing of spike responses, however, it has been previously noted that feedforward inhibition might also be used to control response magnitude, either by preferential amplification of E or I inputs (Douglas et al., 1995) or by extending the range of inputs over which an excitatory population can respond (Pouille et al., 2009). Ahmadian et al. (2013) examined preferential amplification and showed that rate-based networks with an excitatory and inhibitory term that are stable (so that the network does not, e.g., diverge and become epileptic) have regimes of both linearity and sublinearity, although it is not yet clear which of these regimes spiking networks operate in, and which cellular or synaptic parameters affect summation. In the model of Ahmadian et al. (2013), individual cells can be supralinear (Priebe and Ferster, 2008), but when external drive arrives to multiple cells, supralinearity is also seen when recurrent connections are weak and thus excitation and inhibition are not strongly coupled. This may explain why we saw supralinear responses in the model network with the weakest synaptic connectivity (Fig. 5). Analytical work (Ahmadian et al., 2013; Sanzeni et al., 2017) also shows that the range of summation possibilities is limited in the absence of feedforward inhibition. For example, in Ahmadian et al. (2013), their parameters $\Omega_{E}$ and $\Omega_{l}$ describe some summation nonlinearities, and several terms in those parameters disappear when feedforward inhibition (their $g_{l}$ ) is set to zero.

Substantial recurrent intracortical response is elicited by sensory input, with approximately two-thirds of synaptic input after a sensory stimulus arising from recurrent synapses (Li et al., 2013; Lien and Scanziani, 2013). If recurrent connectivity is very strong, previous modeling results (van Vreeswijk and Sompolinsky, 1996; Renart et al., 2010) predict that excitatory and inhibitory populations are forced by the strong coupling to track each others' activity closely, resulting in linear responses. In accord with this prediction about strongly coupled networks, we observed increasing linearity when we increased synaptic strength (Fig. 5) as long as the network remained stable. However, for very strong recurrent connectivity, feedforward connectivity must also be very strong to drive any response (Ahmadian et al., 2013; see also my Fig. 5), which appears nonphysiologic (Li et al., 2013; Lien and Scanziani, 2013). Our simulations use synapses of moderate size (order $1 \mathrm{mV}$ with $2 \%$ sparsity as in Figs. 3, 4, and 6 and Fig. 5, row 4; see Methods), requiring tens of PSPs to combine to produce a spike, as seen in cortical neurons (Barral and Reyes, 2016). These observations suggest that the differences in scaling we observed occur in a range of moderate synaptic strengths (as predicted by Ahmadian et al., 2013): low enough to avoid obligate linearity, and high enough to allow recurrent connections to contribute substantially to network input-output functions.

We found that a network model can link local connectivity to network physiologic responses in ways that might 
be difficult to predict without the model. It has been difficult to measure many of the synapses in a brain volume, but connectomic methods (Briggman et al., 2011; Lee et al., 2016) promise to make such comprehensive synaptic mapping possible even in column-sized volumes of the cortex. Combining approaches for controlling input with methods to measure connectivity will be useful to shed light on an important part of brain computation: the input-output transformations of populations of connected cells.

\section{References}

Adesnik H, Bruns W, Taniguchi H, Huang ZJ, Scanziani M (2012) A neural circuit for spatial summation in visual cortex. Nature 490 : 226-231. CrossRef Medline

Ahmadian Y, Rubin DB, Miller KD (2013) Analysis of the stabilized supralinear network. Neural Computation 25:1994-2037. CrossRef Medline

Atallah BV, Bruns W, Carandini M, Scanziani M (2012) Parvalbuminexpressing interneurons linearly transform cortical responses to visual stimuli. Neuron 73:159-170. CrossRef

Atallah BV, Scanziani M, Carandini M (2014) Atallah et al. reply. Nature 508:E3. CrossRef Medline

Barral J, Reyes AD (2016) Synaptic scaling rule preserves excitatoryinhibitory balance and salient neuronal network dynamics. Nat Neurosci 19:1690-1700. CrossRef

Bonin V, Histed MH, Yurgenson S, Reid RC (2011) Local diversity and fine-scale organization of receptive fields in mouse visual cortex. J Neurosci 31:18506-18521. CrossRef

Bonin V, Mante V, Carandini M (2006) The statistical computation underlying contrast gain control. J Neurosci 26:6346-6353. CrossRef Medline

Braitenberg, V., and Schüz, A. (2001). Cortex: statistics and geometry of neuronal connectivity. In: Neuro- und Sinnesphysiologie, V. Braitenberg, A. Schüz. Berlin, Germany: Springer, 499 pp.

Brette R, Rudolph M, Carnevale T, Hines M, Beeman D, Bower JM, Diesmann M, Morrison A, Goodman PH, Harris FC, et al. (2007) Simulation of networks of spiking neurons: a review of tools and strategies. J Comput Neurosci 23:349-398. CrossRef

Briggman KL, Helmstaedter M, Denk W (2011) Wiring specificity in the direction-selectivity circuit of the retina. Nature 471:183-188. CrossRef Medline

Britten KH, Heuer HW (1999) Spatial summation in the receptive fields of MT neurons. J Neurosci 19:5074-5084. Medline

Brunel N (2000) Dynamics of sparsely connected networks of excitatory and inhibitory spiking neurons. J Comput Neurosci 8:183208. CrossRef

Carandini M, Heeger DJ (2012) Normalization as a canonical neural computation. Nat Rev Neurosci 13:51-62. CrossRef Medline

Chance FS, Abbott LF, Reyes AD (2002) Gain modulation from background synaptic input. Neuron 35:773-782. Medline

Chen JL, Flanders GH, Lee W-CA, Lin WC, Nedivi E (2011) Inhibitory dendrite dynamics as a general feature of the adult cortical microcircuit. J Neurosci 31:12437-12443. CrossRef

Connors BW, Gutnick MJ, Prince DA (1982) Electrophysiological properties of neocortical neurons in vitro. J Neurophysiol 48:13021320. CrossRef

Cruikshank SJ, Lewis TJ, Connors BW (2007) Synaptic basis for intense thalamocortical activation of feedforward inhibitory cells in neocortex. Nat Neurosci 10:462-468. CrossRef

Destexhe A, Paré D (1999) Impact of network activity on the integrative properties of neocortical pyramidal neurons in vivo. J Neurophysiol 81:1531-1547. CrossRef

Destexhe A, Rudolph M, Paré D (2003) The high-conductance state of neocortical neurons in vivo. Nat Rev Neurosci 4:739-751. CrossRef Medline
Douglas RJ, Koch C, Mahowald M, Martin KA, Suarez HH (1995) Recurrent excitation in neocortical circuits. Science 269:981-985. Medline

Douglas RJ, Martin KAC (2004) Neuronal circuits of the neocortex. Annu Rev Neurosci 27:419-451. CrossRef

Freund TF, Martin KA, Soltesz I, Somogyi P, Whitteridge D (1989) Arborisation pattern and postsynaptic targets of physiologically identified thalamocortical afferents in striate cortex of the macaque monkey. J Comp Neur 289:315-336. CrossRef

Gabernet L, Jadhav SP, Feldman DE, Carandini M, Scanziani M (2005) Somatosensory integration controlled by dynamic thalamocortical feed-forward inhibition. Neuron 48:315-327. CrossRef

Garraghty PE, Sur M (1990) Morphology of single intracellularly stained axons terminating in area $3 \mathrm{~b}$ of macaque monkeys. $\mathrm{J}$ Comp Neur 294:583-593. CrossRef

Glickfeld LL, Histed MH, Maunsell JHR (2013) Mouse Primary Visual Cortex Is Used to Detect Both Orientation and Contrast Changes. J Neurosci 33:19416-19422. CrossRef

Gorski JA, Talley T, Qiu M, Puelles L, Rubenstein JLR, Jones KR (2002) Cortical excitatory neurons and glia, but not GABAergic neurons, are produced in the Emx1-expressing lineage. J Neurosci 22:6309-6314.

Häusser M, Spruston N, Stuart GJ (2000) Diversity and dynamics of dendritic signaling. Science 290:739-744. Medline

Histed, M.H., and Maunsell, J.H.R. (2014). Cortical neural populations can guide behavior by integrating inputs linearly, independent of synchrony. Proc Natl Acad Sci USA 111:E178-E187.

Huang X, Elyada YM, Bosking WH, Walker T, Fitzpatrick D (2014) Optogenetic assessment of horizontal interactions in primary visual cortex. J Neurosci 34:4976-4990. CrossRef

Isaacson JS, Scanziani M (2011) How inhibition shapes cortical activity. Neuron 72:231-243. CrossRef Medline

Katz Y, Menon V, Nicholson DA, Geinisman Y, Kath WL, Spruston N (2009) Synapse distribution suggests a two-stage model of dendritic integration in CA1 pyramidal neurons. Neuron 63:171-177. CrossRef

Kawaguchi Y, Kubota Y (1997) GABAergic cell subtypes and their synaptic connections in rat frontal cortex. Cereb Cortex 7:476486. Medline

Kelly RC, Smith MA, Samonds JM, Kohn A, Bonds AB, Movshon JA, Lee TS (2007) Comparison of recordings from microelectrode arrays and single electrodes in the visual cortex. J Neurosci 27: 261-264. CrossRef

Koike H, Mano N, Okada Y, Oshima T (1970) Repetitive impulses generated in fast and slow pyramidal tract cells by intracellularly applied current steps. Exp Brain Res 11:263-281. CrossRef

Lee S-H, Kwan AC, Zhang S, Phoumthipphavong V, Flannery JG, Masmanidis SC, Taniguchi H, Huang ZJ, Zhang F, Boyden ES, et al. (2012) Activation of specific interneurons improves V1 feature selectivity and visual perception. Nature 488:379-383. CrossRef

Lee W-CA, Bonin V, Reed M, Graham BJ, Hood G, Glattfelder K, Reid RC (2016) Anatomy and function of an excitatory network in the visual cortex. Nature 532:370-374. CrossRef Medline

Lefort S, Tomm C, Floyd Sarria J-C, Petersen CCH (2009) The excitatory neuronal network of the $\mathrm{C} 2$ barrel column in mouse primary somatosensory cortex. Neuron 61:301-316. CrossRef

Li Y-t, Ibrahim LA, Liu B-h, Zhang LI, Tao HW (2013) Linear transformation of thalamocortical input by intracortical excitation. Nat Neurosci 16:1324-1330. CrossRef

Lien AD, Scanziani M (2013) Tuned thalamic excitation is amplified by visual cortical circuits. Nat Neurosci 16:1315-1323. CrossRef Medline

Nassi JJ, Avery MC, Cetin AH, Roe AW, Reynolds JH (2015) Optogenetic activation of normalization in alert macaque visual cortex. Neuron 86:1504-1517. CrossRef

Nikolic K, Grossman N, Grubb MS, Burrone J, Toumazou C, Degenaar P (2009) Photocycles of channelrhodopsin-2. Photochem Photobiol 85:400-411. CrossRef Medline 
Olsen SR, Bortone DS, Adesnik H, Scanziani M (2012) Gain control by layer six in cortical circuits of vision. Nature 483:47-52. CrossRef Medline

Phillips EA, Hasenstaub AR (2016) Asymmetric effects of activating and inactivating cortical interneurons. eLife 5:e18383.

Pouille F, Marin-Burgin A, Adesnik H, Atallah BV, Scanziani M (2009) Input normalization by global feedforward inhibition expands cortical dynamic range. Nat Neurosci 12:1577-1585. CrossRef

Priebe NJ, Ferster D (2008) Inhibition, spike threshold, and stimulus selectivity in primary visual cortex. Neuron 57:482-497. CrossRef Medline

Reid RC (2001) Divergence and reconvergence: multielectrode analysis of feedforward connections in the visual system. Prog Brain Res 130:141-154.

Renart A, de la Rocha J, Bartho P, Hollender L, Parga N, Reyes A, Harris KD (2010) The asynchronous state in cortical circuits. Science 327:587-590. CrossRef

Richardson MJE (2004) Effects of synaptic conductance on the voltage distribution and firing rate of spiking neurons. Phys Rev $E$ Stat Nonlin Soft Matter Phys 69:051918. CrossRef Medline

Richardson MJE (2007) Firing-rate response of linear and nonlinear integrate-and-fire neurons to modulated current-based and conductance-based synaptic drive. Phys Rev E Stat Nonlin Soft Matter Phys 76:021919. CrossRef

Rubin DB, Van Hooser SD, Miller KD (2015) The stabilized supralinear network: a unifying circuit motif underlying multi-input integration in sensory cortex. Neuron 85:402-417. CrossRef

Sanzeni, A., Histed, M.H., and Brunel, N. (2017). Emergence of nonlinear computations in spiking neural networks with linear synapses. In 2017 Neuroscience Meeting Planner. Washington, DC: Society for Neuroscience.

Sato TK, Häusser M, Carandini M (2014) Distal connectivity causes summation and division across mouse visual cortex. Nat Neurosci 17:30-32. CrossRef

Silver RA (2010) Neuronal arithmetic. Nat Rev Neurosci 11:474-489. CrossRef Medline
Steriade M, Timofeev I, Grenier F (2001) Natural waking and sleep states: a view from inside neocortical neurons. J Neurophysiol 85:1969-1985. CrossRef Medline

Swadlow HA (2003) Fast-spike interneurons and feedforward inhibition in awake sensory neocortex. Cereb Cortex 13:25-32. CrossRef

Tiesinga P, Fellous J-M, Sejnowski TJ (2008) Regulation of spike timing in visual cortical circuits. Nat Rev Neurosci 9:97-107. CrossRef Medline

Tremblay R, Lee S, Rudy B (2016) GABAergic interneurons in the neocortex: from cellular properties to circuits. Neuron 91:260-292. CrossRef Medline

Van Essen DC, Newsome WT, Maunsell JH (1984) The visual field representation in striate cortex of the macaque monkey: asymmetries, anisotropies, and individual variability. Vision Res 24:429448. CrossRef

van Vreeswijk C, Sompolinsky H (1996) Chaos in neuronal networks with balanced excitatory and inhibitory activity. Science 274: 1724-1726. CrossRef

van Vreeswijk C, Sompolinsky H (1998) Chaotic balanced state in a model of cortical circuits. Neural Computation 10:1321-1371. Medline

Vogels TP, Abbott LF (2005) Signal propagation and logic gating in networks of integrate-and-fire neurons. J Neurosci 25:1078610795. CrossRef Medline

Watakabe A, Ohtsuka M, Kinoshita M, Takaji M, Isa K, Mizukami H, Ozawa K, Isa T, Yamamori T (2015) Comparative analyses of adeno-associated viral vector serotypes 1, 2, 5, 8 and 9 in marmoset, mouse and macaque cerebral cortex. Neurosci Res 93: 144-157. CrossRef

Wilson NR, Runyan CA, Wang FL, Sur M (2012) Division and subtraction by distinct cortical inhibitory networks in vivo. Nature 488:343-348. CrossRef Medline

Wissig SC, Kohn A (2012) The influence of surround suppression on adaptation effects in primary visual cortex. J Neurophysiol 107: 3370-3384. CrossRef

Yamins DLK, DiCarlo JJ (2016) Using goal-driven deep learning models to understand sensory cortex. Nat Neurosci 19:356-365. CrossRef Medline 